\title{
HANDLING MISSING EXTREMES IN TAIL ESTIMATION
}

\author{
HUI XU, RICHARD DAVIS, AND GENNADY SAMORODNITSKY*
}

\begin{abstract}
In some data sets, it may be the case that a portion of the extreme observations are missing. This might arise in cases where the extreme observations are just not available or are imprecisely measured. For example, considering human lifetimes, a topic of recent interest, birth certificates of centenarians may not even exist and many such individuals may not even be included in the data sets that are currently available. In essence, one does not have a clear record of the largest lifetimes of human populations. If there are missing extreme observations, then the assessment of risk can be severely underestimated resulting in rare events occurring more often than originally thought. In concrete terms, this may mean a 500 year flood is in fact a 100 (or even a 20) year flood. In this paper, we present methods for estimating the number of missing extremes together with the tail index associated with tail heaviness of the data. Ignoring one or the other can severely impact the estimation of risk. Our estimates are based on the HEWE (Hill estimate without extremes) of the tail index that adjusts for missing extremes. Based on a functional convergence of this process to a limit process, we consider an asymptotic likelihood-based procedure for estimating both the number of missing extremes and the tail index. We derive the asymptotic distribution of the resulting estimates. By artificially removing segments of extremes in the data, this methodology can be used for assessing the reliability of the underlying assumptions that are imposed on the data.
\end{abstract}

\section{INTRODUCTION}

For modeling heavy-tailed data, the typical operating assumption is that the tails of the common distribution function $F$ are regularly varying. That is,

$$
\frac{\bar{F}(t x)}{\bar{F}(t)} \rightarrow x^{-\alpha}
$$

as $t \rightarrow \infty$ for all $x>0$, where $\alpha>0$ and $\bar{F}(t)=1-F(t)$ is the survival function. The tail index $\alpha$ which governs how heavy the tail is, with smaller $\alpha$ indicating heavier tails, is often the key parameter of interest in applications. The ratio in (1.1) corresponds to the risk probability of $P(X>t x \mid X>t) \sim x^{-\alpha}$ for large $t$ and $x \geq 1$. In fact, the generalized Pareto distribution (GPD), for heavy-tailed distributions essentially originates from this equation:

$$
P(X>t(1+x) \mid X>t) \sim(1+\gamma x)^{-1 / \gamma} \quad x \geq 0
$$

where $\gamma=1 / \alpha>0$ is known as the shape parameter ${ }^{1}$.

1991 Mathematics Subject Classification. Primary 62G32, 60G70.

Key words and phrases. heavy tails, regular variation, missing extremes, tail estimation.

*The corresponding author. This research was partially supported by the ARO grant W911NF-18 -10318 at Cornell University.

${ }^{1}$ The general form of the GPD includes for light- and heavy-tailed has a similar form, see de Haan and Ferreira (2006) 
The most commonly used estimator of $\gamma$ is the Hill estimator defined by

$$
H_{n}(k)=\frac{1}{k} \sum_{i=1}^{k} \log X_{(i)}-\log X_{(k+1)},
$$

where $X_{(1)} \geq X_{(2)} \geq \cdots \geq X_{(n)}$ are the order statistics of an independent and identically distributed (iid) sample $X_{1}, X_{2}, \ldots, X_{n} \sim F$. See Hill (1975) and Drees et al. (2000) for further discussion on this estimator. The Hill estimator is weakly consistent for estimating $\gamma$ provided the number of order statistics $k=k(n)$ used in estimating $\gamma$ satisfies, $k \rightarrow \infty$ and $k / n \rightarrow 0$ as $n \rightarrow \infty$ (see for example de Haan and Ferreira (2006).)

The principal goal of this research is to provide estimates of $\gamma$ and the number of missing extremes in the case when some of the extreme values in the data are missing. As noted in Zou et al. (2019), if some of the extremes in the sample are missing and this is ignored in an estimation procedure, then the tails of the distribution will be underestimated, i.e., they will appear to be lighter than they really are. In terms of risk calculations such as estimating large quantiles, these would be severely underestimated if the estimate of $\gamma$ is too small. With missing extremes, the plot of the Hill estimate as a function of the number $k$ of upper order statistics tends to be increasing and much smoother than without the missing values.

In Zou et al. (2019), the in-degree distribution from a snapshot on October 19, 2012 of the social network Google + was examined. The Google + data, which is owned and operated by Google, consisted of 76,438,791 nodes (registered users) and 1,442,504,499 edges (directed connections). The in-degree of each user is the number of other users following the user. The degree distributions in natural and social networks are often heavy-tailed (see Newman (2010)). Based on the analysis in Zou et al. (2019), it was estimated that around 150 extreme in-degree values were missing, which raises the question of whether these values were excluded from the Google + data set provided to the researchers.

In addition to detecting possible manipulation of data, being aware of the possibility of missing extremes, and developing tools for modeling and analyzing data in the presence of missing extremes, is important in a variety of fields. This includes analysis of natural disasters such as earthquakes, forest fires and floods for which extreme values might be missing due to difficulty in data collection and in an actuarial science context where claims of extremely large amounts might be covered by a reinsurance company and not included in the claims total (Embrechts et al. (1997), Benchaira et al. (2016)). In short, some of the extremes may be just under-reported.

This research builds on the work in Zou et al. (2019), in which an adjusted Hill estimator, called the Hill Estimator Without Extremes (HEWE) is defined that allows for the possibility of missing extreme observations. The HEWE estimator, $H_{n}(\cdot)$ is a process on $(0, \infty)$ that, suitably normalized, converges in law to a Gaussian process $G(\cdot)$ whose covariance function depends on the shape parameter $\gamma$, and on $\delta$, a parameter related to the number of missing extremes. By approximating the distribution of $\left(H_{n}\left(\theta_{1}\right), \ldots, H_{n}\left(\theta_{m}\right)\right)$ for $m$ distinct $\theta$ values, by the distribution of the limiting process, i.e., the distribution of $\left(G\left(\theta_{1}\right), \ldots, G\left(\theta_{m}\right)\right)$, one can use an approximate likelihood procedure to estimate $\gamma$ and $\delta$.

We suggest two implementations of this estimation procedure. The first uses a fixed number of points $\theta$ in which the Hill estimator is evaluated. In the second, where we assume that the observations come from a Pareto sample, the number of points increases to infinity with the sample size. For both estimators we show consistency and asymptotic normality. 
The key to proving our results for the first implementation of the procedure is the strong approximation of the HEWE process $H_{n}(\cdot)$ derived in Zou et al. (2019).

A limitation in our modeling framework is that we assume a consecutive block of the largest observations is missing. A more realistic assumption is that the missing extremes are not necessarily consecutive. A graphical method grounded on our theory for estimating the number of missing extremes is given in Section 5.2. The basic idea is to artificially remove a number of extremes from the observed data. If enough extremes have been removed, then we will have a consecutive number of the largest observations missing from which we can apply our method. The graphical procedure will help us identify the number of extremes to artificially remove from the data in order to perform this second stage estimation.

The paper is organized as follows. In Section 2, we provide some background on the HEWE process $H_{n}(\cdot)$. In Section 3 we describe an approximate asymptotic maximum likelihood estimation procedure of the parameters $\gamma$ and $\delta$ based on the HEWE process, in the case when the Hill estimator is evaluated at a fixed number of points, and establish its consistency and asymptotic normality. In the case when the observations are drawn the Pareto distribution, we describe, in Section 4, another estimation procedure, not directly based on the HEWE process, when the number of points increases to infinity. Section 5 illustrates the methodology via a simulation study. In Section 6, we apply our methodology to three data sets: the well-known Danish fire-insurance claim data, the Google + data, and a a NOAA data set consisting of insurance costs associated with climate and natural disasters in the U.S. The proofs of the results in Sections 3 and 4 are contained in the Appendix (Section 7).

\section{Preliminaries}

In this section we set up the framework for estimating the shape parameter and the number of missing extremes, if any. We assume that the observations come from a sequence $\left\{X_{t}\right\}$ of iid random variables with distribution function $F$ satisfying the regular variation condition (1.1). Let $X_{(1)} \geq X_{(2)} \geq \cdots \geq X_{(n)}$ denote the order statistics of $X_{1}, \ldots, X_{n}$. Given a sequence of integers $k_{n} \rightarrow \infty$ and $k_{n} / n \rightarrow 0$, we view the first $\left\lfloor\delta k_{n}\right\rfloor$ of the order statistics as missing, hence unobservable. Here $\delta \geq 0$, and $\delta=0$ corresponds to the case where no extremes are missing. Hence, with respect to the complete sample, we only have access to the order statistics $X_{\left(\left\lfloor\delta k_{n}\right\rfloor+1\right)}, X_{\left(\left\lfloor\delta k_{n}\right\rfloor+2\right)}, \ldots, X_{(n)}$. The HEWE process is the functional Hill process based on $\left[\theta k_{n}\right\rfloor$ of the observed upper order statistics, given by

$$
H_{n}(\theta)= \begin{cases}\frac{1}{\left\lfloor\theta k_{n}\right\rfloor} \sum_{i=1}^{\left\lfloor\theta k_{n}\right\rfloor} \log X_{\left(\left\lfloor\delta k_{n}\right\rfloor+i\right)}-\log X_{\left(\left\lfloor\delta k_{n}\right\rfloor+\left\lfloor\theta k_{n}\right\rfloor+1\right)}, & \theta \geq 1 / k_{n}, \\ 0, & \theta<1 / k_{n} .\end{cases}
$$

Occasionally, when we want to emphasize the dependence of the HEWE process on $\delta$, we will use the notation $H_{n}(\theta ; \delta)$ instead of $H_{n}(\theta)$.

Of course in practice, we do not know the value of $\delta$ and so $\delta$ is a key parameter of interest to be estimated. If $\delta=0$, then there are no missing extremes and $H_{n}(\cdot)$ is the usual Hill process based on a complete data set.

In Zou et al. (2019), a strong approximation to $H_{n}(\cdot)$ was established under a secondorder regular variation. This condition, which is given in the Appendix (see (7.1)) quantifies the rate of convergence in (1.1). Pareto distributions with tail index $\alpha>0\left(\bar{F}(x)=x^{-\alpha}\right.$ for $x \geq 1$ and zero otherwise) should be viewed as satisfying this condition when the function $A$ 
in the denominator in the left hand side of (7.1) vanishes. In this case (7.2) is automatically satisfied with $\lambda=0$.

We now state the key result (Theorem 2.1(b)) in Zou et al. (2019) that is the basis for our procedures to estimate the shape parameter and the number of the missing extremes, i.e. $\gamma$ and $\delta$.

Theorem 1. Assume that the second-order condition (7.1) holds. Let $k_{n} \rightarrow \infty$ be such that $k_{n} / n \rightarrow 0$ and that (7.2) holds for some $\lambda \in \mathbb{R}$. Then

$$
\sqrt{k_{n}}\left(H_{n}(\cdot ; \delta)-\gamma g_{\delta}(\cdot)\right)-\lambda b_{\delta, \rho}(\cdot) \Rightarrow \gamma G_{\delta}(\cdot)
$$

weakly in $D(0, \infty)$, where

$$
\begin{aligned}
& g_{\delta}(\theta)= \begin{cases}1, & \delta=0, \\
1-(\delta / \theta) \log ((\theta / \delta)+1), & \delta>0,\end{cases} \\
& b_{\delta, \rho}(\theta)= \begin{cases}\frac{1}{1-\rho} \frac{1}{\theta^{\rho}}, & \delta=0, \\
\frac{1+(\theta / \delta) \rho-(\theta / \delta+1)^{\rho}}{(\theta / \delta)(1-\rho) \rho} \frac{1}{(\delta+\theta)^{\rho}}, & \delta>0,\end{cases}
\end{aligned}
$$

and $G_{\delta}(\cdot)$ is a centered Gaussian process with the following representation. Denoting by $W$ the standard Brownian motion,

$$
G_{\delta}(\theta)=\frac{1}{\theta} \int_{\delta}^{\delta+\theta}(1-\delta / x) d W(x), \theta>0
$$

The process $G_{\delta}(\cdot)$ has continuous sample paths and a covariance function given by

$$
\operatorname{Cov}\left(G_{\delta}\left(\theta_{1}\right), G_{\delta}\left(\theta_{2}\right)\right)= \begin{cases}\frac{1}{\theta_{1} \theta_{2}}\left[\theta_{1} \wedge \theta_{2}-2 \delta \log \left(1+\frac{\theta_{1} \wedge \theta_{2}}{\delta}\right)+\frac{\delta\left(\theta_{1} \wedge \theta_{2}\right)}{\delta+\left(\theta_{1} \wedge \theta_{2}\right)}\right], & \delta>0 \\ \frac{1}{\theta_{1} \vee \theta_{2}}, & \delta=0 .\end{cases}
$$

\section{An ALGORITHM FOR ESTIMATING $\gamma$ AND $\delta$}

Throughout this section we fix points $0<\theta_{1}<\cdots<\theta_{m}$. According to Theorem 1 the random vector $\mathbf{H}_{n}=\left(H_{n}\left(\theta_{1}\right), \cdots, H_{n}\left(\theta_{m}\right)\right)^{\prime}$ has, for large $n$, an approximately Gaussian likelihood given by

$$
\sqrt{\frac{k_{n}^{m}}{(2 \pi)^{m} \gamma^{2 m}\left|\Sigma_{m, \delta}\right|}} \exp \left\{-\frac{k_{n}}{2 \gamma^{2}}\left(\mathbf{H}_{n}-\mathbf{g}_{\delta} \gamma-\frac{\lambda \mathbf{b}_{\delta, \rho}}{\sqrt{k_{n}}}\right)^{T} \Sigma_{m, \delta}^{-1}\left(\mathbf{H}_{n}-\mathbf{g}_{\delta} \gamma-\frac{\lambda \mathbf{b}_{\delta, \rho}}{\sqrt{k_{n}}}\right)\right\}
$$

where $\Sigma_{m, \delta}$ is the covariance matrix of the Gaussian vector $\left(G_{\delta}\left(\theta_{1}\right), \cdots, G_{\delta}\left(\theta_{m}\right)\right)^{T}, \mathbf{g}_{\delta}=$ $\left(g_{\delta}\left(\theta_{1}\right), \ldots, g_{\delta}\left(\theta_{m}\right)\right)^{\prime}$, and $\mathbf{b}_{\delta, \rho}=\left(b_{\delta, \rho}\left(\theta_{1}\right), \ldots, b_{\delta, \rho}\left(\theta_{m}\right)\right)^{\prime}$, with $g_{\delta}(\cdot)$ and $b_{\delta, \rho}$ given in $(2.2)$ and (2.3), respectively. Since we are interested in estimating $\gamma$ and $\delta$, while $\rho$ and $\lambda$ are nuisance parameters, we devised a procedure that estimates the parameters of interested while assuming that $\lambda=0$. Note that this eliminates the nuisance parameter $\rho$ as well and leads to a significant simplification in calculations. We will show that the resulting estimators are still consistent and asymptotically normal even if the true value of $\lambda$ is different from 0 . That is, we will maximize the "pseudo-likelihood" function given by

$$
\sqrt{\frac{k_{n}^{m}}{(2 \pi)^{M} \gamma^{2 m}\left|\Sigma_{m, \delta}\right|}} \exp \left\{-\frac{k_{n}}{2 \gamma^{2}}\left(\mathbf{H}_{n}-\mathbf{g}_{\delta} \gamma\right)^{T} \Sigma_{m, \delta}^{-1}\left(\mathbf{H}_{n}-\mathbf{g}_{\delta} \gamma\right)\right\} \text {. }
$$


A change in notation makes the optimization more convenient. Set $\theta_{0}=0$, and denote $T_{n i}=H_{n}\left(\theta_{i}\right)-\left(\theta_{i-1} / \theta_{i}\right) H_{n}\left(\theta_{i-1}\right), \quad h_{\delta i}=g_{\delta, i}-\left(\theta_{i-1} / \theta_{i}\right) g_{\delta, i-1}, i=1, \ldots, m$, with $g_{\delta, i}=g_{\delta}\left(\theta_{i}\right)$, and we interpret $T_{n 1}=H_{n}\left(\theta_{1}\right), h_{\delta 1}=g_{\delta, 1}$. We put $\left.\mathbf{T}_{n}=\left(T_{n 1}, \ldots, T_{n m}\right)\right)^{\prime}$ and $\mathbf{h}_{\delta}=\left(h_{\delta 1}, \ldots, h_{\delta m}\right)^{\prime}$. After some algebraic manipulations, the pseudo-log-likelihood corresponding to (3.2) can be written in the form

$$
C-m \log \gamma+\frac{1}{2} \sum_{i=1}^{m} \log \omega_{i, \delta}-\frac{k_{n}}{2 \gamma^{2}} \sum_{i=1}^{m} \omega_{i, \delta}\left(T_{n i}-\gamma h_{\delta i}\right)^{2}
$$

where

$$
\omega_{i, \delta}=\left\{\begin{array}{ll}
1 /\left(1 / \theta_{i}-\theta_{i-1} / \theta_{i}^{2}\right) & \delta=0 \\
\delta /\left(v\left(\theta_{i} / \delta\right)-\left(\theta_{i-1} / \theta_{i}\right)^{2} v\left(\theta_{i-1} / \delta\right)\right) & \delta>0
\end{array}, \quad v(x)=\frac{1}{x}-\frac{2 \log (1+x)}{x^{2}}+\frac{1}{x(x+1)}\right.
$$

and $C$ is a constant independent of $\gamma$ and $\delta$; see (11) in Zou et al. (2019).

We now separate the notation for the unknown true parameters $\gamma_{0}$ and $\delta_{0}$ in the observed sample from the optimization variables which we continue denoting by $\gamma$ and $\delta$. Let $\mathbf{Y}_{n}=\left(Y_{n 1}, \ldots, Y_{n m}\right)=\gamma_{0}^{-1} \mathbf{T}_{n} \sqrt{k_{n}}-\mathbf{h}_{\delta_{0}} \sqrt{k_{n}}$. This random vector converges weakly to a Gaussian vector with independent components such that, in the limit,

$$
\text { the } i \text { th component is } N\left(\gamma_{0}^{-1} \lambda b_{\delta_{0}, \rho, i}^{*}, 1 / \omega_{i, \delta_{0}}\right) \text {, }
$$

where

$$
b_{\delta_{0}, \rho, i}^{*}=b_{\delta_{0}, \rho}\left(\theta_{i}\right)-\frac{\theta_{i-1}}{\theta_{i}} b_{\delta_{0}, \rho}\left(\theta_{i-1}\right), i=1, \ldots, m ;
$$

see Zou et al. (2019). Then the pseudo-log-likelihood becomes

$$
C-m \log \gamma+\frac{1}{2} \sum_{i=1}^{m} \log \omega_{i, \delta}-\frac{1}{2 \gamma^{2}} \sum_{i=1}^{m} \omega_{i, \delta}\left(\gamma_{0} Y_{n i}-\left(\gamma h_{\delta i}-\gamma_{0} h_{\delta_{0} i}\right) \sqrt{k_{n}}\right)^{2} .
$$

Since $C$ is independent of $\gamma$ and $\delta$, we ignore this term and optimize the function

$$
L_{n}(\gamma, \delta)=2 m \log \gamma-\sum_{i=1}^{m} \log \omega_{i, \delta}+\frac{1}{\gamma^{2}} \sum_{i=1}^{m} \omega_{i, \delta}\left(\gamma_{0} Y_{n i}-\sqrt{k_{n}}\left(\gamma h_{\delta i}-\gamma_{0} h_{\delta_{0}, i}\right)\right)^{2} .
$$

The main result of this section, Theorem 3.1 below, proves the consistency and the asymptotic normality of the estimators obtained by minimizing the function $L_{n}$. It applies only in the case when $\delta_{0}>0$, i.e. when some extremes are missing. One can interpret the case when no extremes are missing as corresponding to a small but positive value of $\delta_{0}$. We will assume that the true value of the parameters, $\left(\gamma_{0}, \delta_{0}\right)$, belong to the interior $\Theta^{o}$ of a known compact set $\Theta=\left[m_{1}, M_{1}\right] \times\left[m_{2}, M_{2}\right] \subset(0, \infty)^{2}$, and compute our estimator via

$$
(\hat{\gamma}, \hat{\delta})=\arg \min _{(\gamma, \delta) \in \Theta} L_{n}(\gamma, \delta) \text {. }
$$

The following quantities will be used in the statement of our main results. Let

$$
b_{m}=\sum_{i=1}^{m} \omega_{i, \delta_{0}} h_{\delta_{0}, i}^{2}, \quad c_{m}=\sum_{i=1}^{m} \omega_{i, \delta_{0}}\left(h_{\delta_{0}, i}^{\prime}\right)^{2}, \quad d_{m}=\sum_{i=1}^{m} \omega_{i, \delta_{0}} h_{\delta_{0}, i} h_{\delta_{0}, i}^{\prime} .
$$

where $h_{\delta_{0}, i}^{\prime}=\left.\frac{\mathrm{d} h_{\delta, i}}{\mathrm{~d} \delta}\right|_{\delta=\delta_{0}}$. 
Theorem 3.1. Assume that the second-order condition (7.1) holds. Let $k_{n} \rightarrow \infty$ be such that $k_{n} / n \rightarrow 0$ and that (7.2) holds for some $\lambda \in \mathbb{R}$. Suppose that $\delta_{0}>0$, that $\left(\gamma_{0}, \delta_{0}\right) \in \Theta^{o}$, and let $m \geq 2$ be fixed. Then the optimization problem (3.5) has a unique solution $(\hat{\gamma}, \hat{\delta})$ with probability increasing to 1 as $n \rightarrow \infty$. This solution is a weakly consistent estimator of $\left(\gamma_{0}, \delta_{0}\right)$, and

$$
\left(\sqrt{k_{n}}\left(\hat{\gamma}-\gamma_{0}\right), \sqrt{k_{n}}\left(\hat{\delta}-\delta_{0}\right)\right) \Rightarrow N\left(.5 \Gamma_{m}^{-1} \mathbf{a}, \Gamma_{m}^{-1}\right)
$$

as $n \rightarrow \infty$, where in the notation of (3.6),

$$
\Gamma_{m}=\left[\begin{array}{cc}
\frac{b_{m}}{\gamma_{0}^{2}} & \frac{d_{m}}{\gamma_{0}} \\
\frac{d_{m}}{\gamma_{0}} & c_{m}
\end{array}\right] \quad \text { and } \quad \Gamma_{m}^{-1}=\left[\begin{array}{cc}
\frac{\gamma_{0}^{2} c_{m}}{b_{m} c_{m}-d_{m}^{2}} & -\frac{\gamma_{0} d_{m}}{b_{m} c_{m}-d_{m}^{2}} \\
-\frac{\gamma_{0} d_{m}}{b_{m} c_{m}-d_{m}^{2}} & \frac{b_{m}}{b_{m} c_{m}-d_{m}^{2}}
\end{array}\right],
$$

and $\mathbf{a}=\left(a_{1}, a_{2}\right)^{T}$ with

$$
a_{1}=2 \gamma_{0}{ }^{-2} \lambda \sum_{i=1}^{m} \omega_{i, \delta_{0}} h_{\delta_{0}, i} b_{\delta_{0}, \rho, i}^{*}, \quad a_{2}=2 \gamma_{0}{ }^{-1} \lambda \sum_{i=1}^{m} \omega_{i, \delta_{0}} h_{\delta_{0}, i}^{\prime} b_{\delta_{0}, \rho, i}^{*} .
$$

A part of the claim of Theorem 3.1 is the non-singularity of the matrix $\Gamma_{m}$, which we will establish below. It is interesting to compare the performance of the estimator $\hat{\gamma}$ of the shape parameter given in the theorem with the limiting variance of the plain Hill estimator of the shape parameter. Of course, the Hill estimator is only consistent when there are no missing extremes, i.e. when $\delta_{0}=0$, whereas Theorem 3.1 only applies in the case $\delta_{0}>0$. However, the comparison is instructive if one takes the limiting distribution in Theorem 3.1 and considers the situation when $\delta_{0} \rightarrow 0$. It would not be surprising to expect a loss in efficiency, in the sense that having to estimate both $\gamma$ and $\delta$ may lead to higher bias and/or higher variance of the estimator of the shape parameter from Theorem 3.1 in comparison with the Hill estimator which does not need to estimate $\delta$. However, it turns that there is no loss of efficiency, after all. We have the following proposition.

Proposition 3.1. Suppose that $\delta_{0}>0$ and let $m \geq 2$ be fixed. Then the matrix $\Gamma_{m}$ is is invertible and, as $\delta_{0} \downarrow 0$, the parameters of the Gaussian limit of $\sqrt{k_{n}}\left(\hat{\gamma}-\gamma_{0}\right)$ satisfy

$$
\begin{aligned}
& \text { the mean converges to } \frac{\lambda}{1-\rho} \frac{\theta_{m}^{1-\rho}-\theta_{1}^{1-\rho}}{\theta_{m}-\theta_{1}}, \\
& \text { the variance converges to } \frac{\gamma_{0}^{2}}{\theta_{m}-\theta_{1}} \text {. }
\end{aligned}
$$

Recall that under the assumptions(7.1) and (7.2) the Hill estimator of $\gamma$ satisfies

$$
\sqrt{k_{n}}\left(H_{n}\left(k_{n}\right)-\gamma_{0}\right) \Rightarrow N\left(\lambda /(1-\rho), \gamma_{0}^{2}\right) ;
$$

see e.g. Theorem 3.2.5 in de Haan and Ferreira (2006).

The significance of (3.10) becomes clear when we let $\theta_{m}=1$ and choose $\theta_{0}$ close to 0 , in which case the bias and the variance become the same as those in (3.11).

The proof of Theorem 3.1 (including the invertibility of the matrix $\Gamma_{m}$ ) will follow from a sequence of lemmas, whose proofs are given in the appendix. We start with the asymptotic behavior of the gradient of the function $L_{n}$ evaluated at the true values of the parameters. 
Lemma 3.1. Suppose that $\delta_{0}>0$ and let $m \geq 2$ be fixed. Then

$$
\left(\frac{\partial_{1} L_{n}\left(\gamma_{0}, \delta_{0}\right)}{\sqrt{k_{n}}}, \frac{\partial_{2} L_{n}\left(\gamma_{0}, \delta_{0}\right)}{\sqrt{k_{n}}}\right) \Rightarrow N\left(-\mathbf{a}, 4 \Gamma_{m}\right) .
$$

Next, we address the asymptotic behavior of the Hessian matrix

$$
\mathcal{H}_{n}(\gamma, \delta)=\left[\begin{array}{cc}
\partial_{1}^{2} L_{n}(\gamma, \delta) & \partial_{1} \partial_{2} L_{n}(\gamma, \delta) \\
\partial_{1} \partial_{2} L_{n}(\gamma, \delta) & \partial_{2}^{2} L_{n}(\gamma, \delta)
\end{array}\right]
$$

of the function $L_{n}$ evaluated at any weakly consistent estimator of the true values.

Lemma 3.2. Suppose that $\delta_{0}>0$ and let $m \geq 2$ be fixed. If $(\tilde{\gamma}, \tilde{\delta}) \stackrel{P}{\rightarrow}\left(\gamma_{0}, \delta_{0}\right)$ then $k_{n}^{-1} \mathcal{H}_{n}(\tilde{\gamma}, \tilde{\delta}) \stackrel{P}{\rightarrow} 2 \Gamma_{m}$

The next lemma proves the weak consistency of our estimator.

Lemma 3.3. Suppose that $\delta_{0}>0$ and let $m \geq 2$ be fixed. Then the optimization problem (3.5) has a unique solution $(\hat{\gamma}, \hat{\delta})$ with probability increasing to 1 as $n \rightarrow \infty$ and

$$
(\hat{\gamma}, \hat{\delta}) \stackrel{P}{\rightarrow}\left(\gamma_{0}, \delta_{0}\right)
$$

Proof of Theorem 3.1. The Taylor expansion of the gradient of $L_{n}$ around the true values of the parameter tells us that

$$
\left(\frac{\partial_{1} L_{n}(\hat{\gamma}, \hat{\delta})}{\sqrt{k_{n}}}, \frac{\partial_{2} L_{n}(\hat{\gamma}, \hat{\delta})}{\sqrt{k_{n}}}\right)^{T}=\left(\frac{\partial_{1} L_{n}\left(\gamma_{0}, \delta_{0}\right)}{\sqrt{k_{n}}}, \frac{\partial_{2} L_{n}\left(\gamma_{0}, \delta_{0}\right)}{\sqrt{k_{n}}}\right)^{T}+\frac{\mathcal{H}_{n}(\bar{\gamma}, \bar{\delta})}{\sqrt{k_{n}}}\left(\hat{\gamma}-\gamma_{0}, \hat{\delta}-\delta_{0}\right)^{T}
$$

for some $(\bar{\gamma}, \bar{\delta})$ between $(\hat{\gamma}, \hat{\delta})$ and $\left(\gamma_{0}, \delta_{0}\right)$. By Lemma 3.3 , with probability increasing to 1 , the infimum in (3.5) is achieved in the interior of the set $\Theta$, and on that event $\left(\partial_{1} L_{n}(\hat{\gamma}, \hat{\delta}), \partial_{2} L_{n}(\hat{\gamma}, \hat{\delta})\right)^{T}=(0,0)^{T}$. Therefore, the relation

$$
-\left(\frac{\partial_{1} L_{n}\left(\gamma_{0}, \delta_{0}\right)}{\sqrt{k_{n}}}, \frac{\partial_{2} L_{n}\left(\gamma_{0}, \delta_{0}\right)}{\sqrt{k_{n}}}\right)^{T}=\frac{\mathcal{H}_{n}(\bar{\gamma}, \bar{\delta})}{\sqrt{k_{n}}}\left(\hat{\gamma}-\gamma_{0}, \hat{\delta}-\delta_{0}\right)^{T}
$$

also holds on an event whose probability increases to 1 . Lemma 3.3, also implies that $(\bar{\gamma}, \bar{\delta}) \stackrel{P}{\rightarrow}\left(\gamma_{0}, \delta_{0}\right)$, so the claim of the theorem follows from Lemma 3.1 and Lemma 3.2.

\section{Estimating $\gamma$ And $\delta$ in a Pareto Sample}

In this section we assume that the observations $X_{1}, X_{2}, \ldots$ follow the Pareto distribution $\bar{F}(x)=x^{-\alpha}$ for $x \geq 1$. In this case the exact distribution of the order statistics is available, so we will not need to rely as much on the asymptotic normality of $H_{n}$ in Theorem 1. Unlike the algorithm of the previous section, we will now use $k_{n}$ equally spaced points $\theta_{i, n}=\varepsilon+i / k_{n}, 1 \leq i \leq k_{n}$, for some $\varepsilon>0$.

Let, once again, $X_{(1)}>X_{(2)}>\cdots>X_{(n)}$ be the order statistics from the sample $X_{1}, \ldots, X_{n}$. Then

$$
\left(X_{(1)}, \cdots, X_{(n)}\right) \stackrel{d}{=}\left(\left(\frac{S_{n+1}}{S_{1}}\right)^{1 / \gamma}, \cdots,\left(\frac{S_{n+1}}{S_{n}}\right)^{1 / \gamma}\right)
$$


see e.g. Corollary 1.6.9 of Reiss (1989). Here $S_{i}=\sum_{j=1}^{i} E_{j}$, with $\left(E_{j}\right)$ iid standard exponential random variables. It follows that the HEWE process satisfies

$$
\left(H_{n}\left(\theta_{i, n}\right), i=1, \ldots, k_{n}\right) \stackrel{d}{=}\left(\frac{\gamma}{\left\lfloor\theta_{i, n} k_{n}\right\rfloor} \sum_{i=1}^{\left\lfloor\theta_{i, n} k_{n}\right\rfloor} \log \left(\frac{S_{\left\lfloor\delta k_{n}\right\rfloor+\left\lfloor\theta_{i, n} k_{n}\right\rfloor+1}}{S_{\left\lfloor\delta k_{n}\right\rfloor+i}}\right), i=1, \ldots, k_{n}\right) .
$$

Since $E_{i}^{*}=: i \log \left(S_{i+1} / S_{i}\right), i=1, \ldots, n$, are also iid standard exponential random variables, a bit of algebra shows that

$$
\left(H_{n}\left(\theta_{i, n}\right), i=1, \ldots, k_{n}\right) \stackrel{d}{=}\left(\frac{\gamma}{\left\lfloor\theta_{i, n} k_{n}\right\rfloor} \sum_{i=\left\lfloor\delta k_{n}\right\rfloor+1}^{\left\lfloor\delta k_{n}\right\rfloor+\left\lfloor\theta_{i, n} k_{n}\right\rfloor}\left(1-\frac{\left\lfloor\delta k_{n}\right\rfloor}{i}\right) E_{i}^{*}, i=1, \ldots, k_{n}\right) .
$$

Denote now $\xi_{i, n}=H_{n}\left(\theta_{i, n}\right)-\left\lfloor\theta_{i-1, n} k_{n}\right\rfloor H_{n}\left(\theta_{i-1, n}\right) /\left\lfloor\theta_{i, n} k_{n}\right\rfloor, i=1, \ldots, k_{n}$ and set $\theta_{0, n}=0$. Since for any $i \geq 2$ we have $\left\lfloor\theta_{i, n} k_{n}\right\rfloor-\left\lfloor\theta_{i-1, n} k_{n}\right\rfloor=1$, it follows that

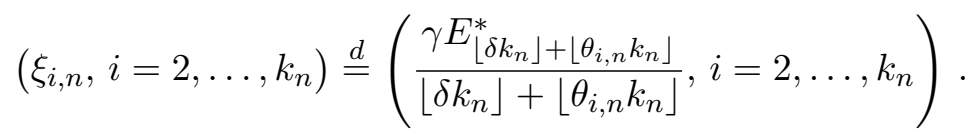

We wish to perform an MLE procedure based on the joint density of $\left(\xi_{1, n}, \cdots, \xi_{k_{n}, n}\right)$. The joint density of $\left(\xi_{2, n}, \cdots, \xi_{k_{n}, n}\right)$ can be read off (4.1). Since these random variables are independent of $\xi_{1, n}$, we will construct a mixed likelihood function by combining the exact joint density of $\left(\xi_{2, n}, \cdots, \xi_{k_{n}, n}\right)$ with the asymptotic normal density for $\xi_{1, n}$ given in (3.2) with $m=1$ and $\theta_{1}=\theta_{1, n}$. We simplify the resulting expression by dropping the rounding (replacing $\left\lfloor\delta k_{n}\right\rfloor$ by $\delta k_{n}$ and $\left\lfloor\theta_{i, n} k_{n}\right\rfloor$ by $\theta_{i, n} k_{n}$ as needed) Taking the logarithm of the result multiplied by -2 gives us the following function of $\gamma$ and $\delta$ (a pseudo-log-likelihood function, modulo additive constants not depending on $\gamma$ or $\delta$ ), to be optimized:

$$
\begin{aligned}
L_{n}(\gamma, \delta) & =2 \log \gamma-\log \omega_{1, \delta}-2 \sum_{i=2}^{k_{n}} \log \left(\frac{\delta+\theta_{i, n}}{\gamma}\right) \\
& +\frac{k_{n} \omega_{1, \delta}}{\gamma^{2}}\left(\xi_{1, n}-\gamma g_{\delta, 1}\right)^{2}+\frac{2 k_{n}}{\gamma} \sum_{i=2}^{k_{n}}\left(\delta+\theta_{i, n}\right) \xi_{i, n} .
\end{aligned}
$$

As in the previous section, we separate the notation for the unknown true parameters $\gamma_{0}$ and $\delta_{0}$ from the optimization variables $\gamma$ and $\delta$. Denote $\eta_{n}=\gamma_{0}^{-1} \xi_{1, n} \sqrt{k_{n}}-g_{\delta_{0}, 1} \sqrt{k_{n}}$ and $Z_{i, n}=k_{n}\left(\delta_{0}+\theta_{i, n}\right) \xi_{i, n} / \gamma_{0}$, and notice that these independent random variables satisfy $\eta_{n} \stackrel{d}{\rightarrow} N\left(0,1 / \omega_{1, \delta_{0}}\right)$ and each $Z_{i, n}$ converges to a standard exponential random variable. With this notation the function to be minimized becomes

$$
\begin{aligned}
L_{n}(\gamma, \delta)= & 2 k_{n} \log \gamma-\log \omega_{1, \delta}-2 \sum_{i=2}^{k_{n}} \log \left(\delta+\theta_{i, n}\right)+\frac{\omega_{1, \delta}}{\gamma^{2}}\left(\gamma_{0} \eta_{n}-\sqrt{k_{n}}\left(\gamma g_{\delta, 1}-\gamma_{0} g_{\delta_{0}, 1}\right)\right)^{2} \\
& +\frac{2 \gamma_{0}}{\gamma} \sum_{i=2}^{k_{n}} \frac{\left(\delta+\theta_{i, n}\right) Z_{i, n}}{\delta_{0}+\theta_{i, n}} .
\end{aligned}
$$

As in the previous section we will assume that the true value of the parameters, $\left(\gamma_{0}, \delta_{0}\right)$, belong to the interior $\Theta^{o}$ of a known compact set $\Theta=\left[m_{1}, M_{1}\right] \times\left[m_{2}, M_{2}\right] \subset(0, \infty)^{2}$, and compute our estimator via (3.5), this time using $L_{n}$ in (4.2).

The following is the main result of this section. 
Theorem 4.1. Suppose that the observations $X_{1}, X_{2}, \ldots$ follow the Pareto distribution. Let $k_{n} \rightarrow \infty$ be such that $k_{n} / n \rightarrow 0$. Suppose that $\delta_{0}>0$, that $\left(\gamma_{0}, \delta_{0}\right) \in \Theta^{o}$, and let $\theta_{i, n}=\varepsilon+i / k_{n}, 1 \leq i \leq k_{n}$ for some $\varepsilon>0$. Then the optimization problem (3.5) has a unique solution $(\hat{\gamma}, \hat{\delta})$ with probability increasing to 1 as $n \rightarrow \infty$. This solution is a weakly consistent estimator of $\left(\gamma_{0}, \delta_{0}\right)$, and

$$
\left(\sqrt{k_{n}}\left(\hat{\gamma}-\gamma_{0}\right), \sqrt{k_{n}}\left(\hat{\delta}-\delta_{0}\right)\right) \Rightarrow N\left(0, \Gamma_{\infty}^{-1}\right)
$$

where

$$
\Gamma_{\infty}=\Gamma_{1}+\left[\begin{array}{cc}
\gamma_{0}^{-2} & -\gamma_{0}^{-1} \log \left(1+1 /\left(\delta_{0}+\varepsilon\right)\right) \\
-\gamma_{0}^{-1} \log \left(1+1 /\left(\delta_{0}+\varepsilon\right)\right) & \left(\delta_{0}+\varepsilon\right)^{-1}\left(\delta_{0}+\varepsilon+1\right)^{-1}
\end{array}\right]
$$

and $\Gamma_{1}$ is as in (3.8) with $m=1$ and $\theta_{1}=\varepsilon$. The matrix $\Gamma_{\infty}$ is invertible with

$$
\Gamma_{\infty}^{-1}=\Delta^{-1}\left[\begin{array}{cc}
\gamma_{0}^{2}\left(c_{1}+\left(\delta_{0}+\varepsilon\right)^{-1}\left(\delta_{0}+\varepsilon+1\right)^{-1}\right) & \gamma_{0}\left(-d_{1}+\log \left(1+1 /\left(\delta_{0}+\varepsilon\right)\right)\right) \\
\gamma_{0}\left(-d_{1} \log \left(1+1 /\left(\delta_{0}+\varepsilon\right)\right)\right) & b_{1}+1
\end{array}\right]
$$

where $\Delta=\left(b_{1}+1\right)\left(c_{1}+\left(\delta_{0}+\varepsilon\right)^{-1}\left(\delta_{0}+\varepsilon+1\right)^{-1}\right)-\left(d_{1}-\log \left(1+1 /\left(\delta_{0}+\varepsilon\right)\right)\right)^{2}$.

The structure of proof of Theorem 4.1 is nearly identical to that of Theorem 3.1, with Lemmas 3.1-3.3 replaced by their counterparts, Lemmas 4.1-4.3. Once again, we start with the asymptotic behavior of the gradient of the function $L_{n}$ evaluated at the true values of the parameters.

Lemma 4.1. Suppose $\delta_{0}>0$ and $\theta_{i, n}=\varepsilon+i / k_{n}, 1 \leq i \leq k_{n}$. Then

$$
\left(\frac{\partial_{1} L_{n}\left(\gamma_{0}, \delta_{0}\right)}{\sqrt{k_{n}}}, \frac{\partial_{2} L_{n}\left(\gamma_{0}, \delta_{0}\right)}{\sqrt{k_{n}}}\right) \Rightarrow N\left(0,4 \Gamma_{\infty}\right) .
$$

As before, we proceed with the asymptotic behavior of the Hessian matrix (3.12) of the function $L_{n}$ evaluated at a weakly consistent estimator of the true values.

Lemma 4.2. Suppose that $\delta_{0}>0$ and let $\theta_{i, n}=\varepsilon+i / k_{n}, i=1, \ldots, k_{n}$. If $(\tilde{\gamma}, \tilde{\delta}) \stackrel{P}{\rightarrow}\left(\gamma_{0}, \delta_{0}\right)$ then $k_{n}^{-1} \mathcal{H}_{n}(\tilde{\gamma}, \tilde{\delta}) \stackrel{P}{\rightarrow} 2 \Gamma_{\infty}$.

The final lemma, once again, proves the weak consistency.

Lemma 4.3. Suppose that $\delta_{0}>0$ and let $\theta_{i, n}=\varepsilon+i / k_{n}, i=1, \ldots, k_{n}$. Then the optimization problem (3.5) has a unique solution $(\hat{\gamma}, \hat{\delta})$ with probability increasing to 1 as $n \rightarrow \infty$ and

$$
(\hat{\gamma}, \hat{\delta}) \stackrel{P}{\rightarrow}\left(\gamma_{0}, \delta_{0}\right)
$$

Proof of Theorem 4.1. One can use an argument identical to that in the proof of Theorem 3.1 , hence only the invertibility of $\Gamma_{\infty}$ needs to be shown. Since $\Gamma_{1}$ is nonnegative definite, we only have to check that the second matrix in $\Gamma_{\infty}$ has a positive determinant. However, by Jensen's inequality,

$$
\left(\delta_{0}+\varepsilon\right)^{-1}\left(\delta_{0}+\varepsilon+1\right)^{-1}-\left(\log \left(1+\frac{1}{\delta_{0}+\varepsilon}\right)\right)^{2}
$$




$$
=\int_{\varepsilon}^{1+\varepsilon} \frac{1}{\left(\delta_{0}+x\right)^{2}} d x-\left(\int_{\varepsilon}^{1+\varepsilon} \frac{1}{\delta_{0}+x} d x\right)^{2}>0,
$$

as required.

Remark 4.1. It is elementary to check that the entry in the upper left corner of the matrix $\Gamma_{\infty}^{-1}$ converges, as $\delta_{0} \rightarrow 0$, to $\gamma_{0}^{2}$. This is the same somewhat surprising lack of efficiency lost we have seen in Proposition 3.1.

\section{Simulation Results}

5.1. Estimation of $\delta$ and $\gamma$. In this section, we compare the performance of the estimation procedures described in Sections 3 and 4 on simulated data. As a test data set, we generate $n=5000$ observations from a Pareto distribution $(F(x)=1-1 / x, x \geq 1)$ and from a standard Fréchet distribution $\left(F(x)=\exp \left\{-x^{-1}\right\}, x \geq 0\right)$. We chose $k_{n}=200$ in all cases, and use 3 different values of $\delta_{0}=0.1,0.2,0.5$ corresponding to the top 20, 40 and 100 missing extremes, respectively. For the estimation method of Section 3, we chose $m=10$ distinct $\theta$ 's with $\theta_{i}=i / 10, i=1, \ldots, 10$, and minimized the pseudo-likelihood function given in (3.5) with respect to $\delta$ and $\gamma$. This was repeated 1000 times and the summary statistics (means and standard deviations) are given in Table 1 (Pareto) and Table 2 (Fréchet) corresponding to the columns labeled $\hat{\delta}_{a}$ and $\hat{\gamma}_{a}$. Notice that both the bias and standard deviation of $\hat{\delta}_{a}$ increase with $\delta_{0}$ where the latter increases at a rate that is roughly proportionally to $\delta_{0}$.

We also used the estimation procedure of Section $4\left(m=k_{n}, \varepsilon=1 / 200\right)$ in which the objective function in (4.2) was minimized. This procedure was applied to the same Pareto and Fréchet generated data as before, even though, in theory, the method was introduced only for Pareto samples. The results are also summarized in Tables 1 and 2 using the labels $\hat{\delta}_{b}$ and $\hat{\gamma}_{b}$. The bias for $\hat{\delta}_{b}$ is considerably smaller than that for $\hat{\delta}_{a}$ in most cases (even in the Fréchet samples). The standard deviations were also a bit smaller in all cases. On the other hand the biases for $\hat{\gamma}_{b}$ were similar to those for $\hat{\gamma}_{a}$, but in all cases the standard deviation was a touch smaller. This may not be too surprising since these estimates are based on more $\theta_{i}$. The asymptotic standard deviations for both the two estimates using $m=10$ and $m=k_{n}$ can be computed using the formulae in (3.8) and (4.3), respectively, were all smaller than their finite sample counterparts. For example, in the Pareto case for $\hat{\delta}_{a}$ the asymptotic standard errors for $\delta_{0}=0.1,0.2,0.5$ were $0.047,0.083,0.219$, respectively.

It is worth emphasizing again that the two estimation procedures based on $m=10$ and $m=k_{n}$ generally performed well for the Fréchet case even though our theory for $m=k_{n}$ is not directly applicable to this case. Interestingly, the biases and standard deviations were generally smaller in the Fréchet case in comparison with the Pareto case, across the range of parameter values and the two estimation procedures. The histograms of the estimates leading to $\hat{\delta}_{a}$ in Table 2 corresponding to $\delta_{0}=0.1$ and 0.5 are displayed in Figure 1 . Notice the long right tails. This is more pronounced in the $\delta_{0}=0.5$ case, which is due in part to having fewer of the most extreme observations to estimate tail parameters.

Tables 1 and 2 also contain two additional columns showing correlations (both sample

and asymptotic based on (3.8) and (4.3)) between $\hat{\delta}_{a}$ and $\hat{\gamma}_{a}$ and between $\hat{\delta}_{b}$ and $\hat{\gamma}_{b}$. There is good agreement between the sample and asymptotic correlations and all are large (close to 1). That means that in the two-dimensional optimization likelihood procedure moderate errors in estimating $\gamma_{0}$ lead to significant errors in estimating $\delta_{0}$ because of the large standard errors of the estimates of $\delta_{0}$. It turns out, however, that fixing $\gamma$, the one-dimensional 
likelihood optimization procedures for $\delta$ has less variability and is, moreover, fairly robust to a mild misspecification of $\gamma$. We exploit this fact in the sequel.

TABLE 1. Pareto distribution, $n=5000, k_{n}=200$

\begin{tabular}{llllllll}
\hline & \multicolumn{2}{c}{$\hat{\delta}_{a}$} & \multicolumn{2}{c}{$\hat{\gamma}_{a}$} & \multicolumn{2}{c}{$\rho_{\hat{\delta}_{a}, \hat{\gamma}_{a}}$} & \multicolumn{2}{c}{$\hat{\delta}_{b}$} & \multicolumn{2}{c}{$\hat{\gamma}_{b}$} & \multicolumn{2}{c}{$\rho_{\hat{\delta}_{b}, \hat{\gamma}_{b}}$} \\
$\delta_{0}$ & mean $(\mathrm{sd})$ & mean & $(\mathrm{sd})$ & corr $($ asy $)$ & mean $(\mathrm{sd})$ & mean $(\mathrm{sd})$ & corr $($ asy $)$ \\
\hline 0.1 & $0.113(0.057)$ & $1.015(0.143)$ & $0.858(0.829)$ & $0.104(0.049)$ & $1.006(0.129)$ & $0.841(0.796)$ \\
0.2 & $0.222(0.104)$ & $1.025(0.187)$ & $0.915(0.894)$ & $0.207(0.096)$ & $1.010(0.177)$ & $0.915(0.878)$ \\
0.5 & $0.547(0.285)$ & $1.040(0.309)$ & $0.965(0.956)$ & $0.515(0.254)$ & $1.014(0.282)$ & $0.962(0.951)$ \\
\hline
\end{tabular}

TABLE 2. Fréchet distribution, $n=5000, k_{n}=200$

\begin{tabular}{|c|c|c|c|c|c|c|}
\hline & $\hat{\delta}_{a}$ & $\hat{\gamma}_{a}$ & $\rho_{\hat{\delta}_{a}}$ & $\hat{\delta}_{b}$ & $\hat{\gamma}_{b}$ & $\rho_{\hat{\delta}_{t}}$ \\
\hline$\delta_{0}$ & mean $(\mathrm{sd})$ & mean & corr (asy) & mean (sd) & mean (sd) & corr (asy) \\
\hline 0.1 & $0.106(0.050$ & $0.992(0.130)$ & $0.829(0.829$ & $0.101(0.045)$ & $0.988(0.122)$ & $0.826(0.796)$ \\
\hline 0.2 & 0.2 & $0.993(0$ & $0.906(0$ & $(0$. & $(0$. & $0.904(0$. \\
\hline 0.5 & $0.535(0.287)$ & $1.011(0.300)$ & $0.961(0.956)$ & $0.502(0.252)$ & $0.985(0.274)$ & $0.961(0.951)$ \\
\hline
\end{tabular}

5.2. Graphical methods for estimating the number of missing extremes. Although our estimation procedure assumes that $\delta_{0} k_{n}$ of the largest extremes are missing, in practice, it might be more likely that missing extremes, if any exist, do not occur consecutively from the largest. Our method can still be used to estimate the total number of missing extremes. For example if there are 10 missing extremes scattered among the largest 50, we can artificially remove the largest 40 extremes from the data set. The altered data set can then be viewed as having 50 consecutive missing extremes and our estimation procedure for estimating the number of missing extremes is applicable. If the resulting estimate is near 50, as it should be, then since we know that 40 have been artificially removed, we would be able to recover an estimate of the number of original missing extremes, even when non-consecutive. We use a graphical procedure to give an idea of how this works.

For a given data set with $\delta_{0} k_{n}$ missing observations among the largest $\left(\delta_{0}+\delta^{\dagger}\right) k_{n}$ observations, we remove for each $i=1,2, \ldots, k_{n}$, the $i$ largest observations (this corresponds to $\left.\delta=i / k_{n}\right)$ from the observed data and produce estimates $\hat{\delta}$. Once $\delta \geq \delta^{\dagger}$, then the $\left(\delta+\delta_{0}\right) k_{n}$ largest extremes $\left(\delta_{0} k_{n}\right.$ unknown to us plus $\delta k_{n}$ artificially removed) are now missing. The estimates $\hat{\delta}$ for $\delta \geq \delta^{\dagger}$ should be approximately linear in $\delta$ with a slope close to 1 . The idea is then to pick off the threshold for which the plot of $\hat{\delta}$ vs. $\delta$ becomes linear for $\delta$ larger than that threshold. This value is then identified as $\delta^{\dagger}$ and the difference $\hat{\delta}-\delta^{\dagger}$ is then an estimate of $\delta_{0}$. Once we have estimated $\delta^{\dagger}$, if desired, we can remove the largest $\delta^{\dagger} k_{n}$ observations and re-apply our estimation procedure to provide updated estimates of $\delta_{0}$ and $\gamma_{0}$.

We illustrate this procedure with several simulation examples under three different scenarios: (i) no missing extremes, (ii) the upper $\delta_{0} k_{n}$ are missing and (iii) the $\delta_{0} k_{n}$ missing extremes are not consecutive upper extremes. The setup for this simulation is similar to that of Section 5.1. Samples of size $n=5000$ are generated from both Pareto and Fréchet 

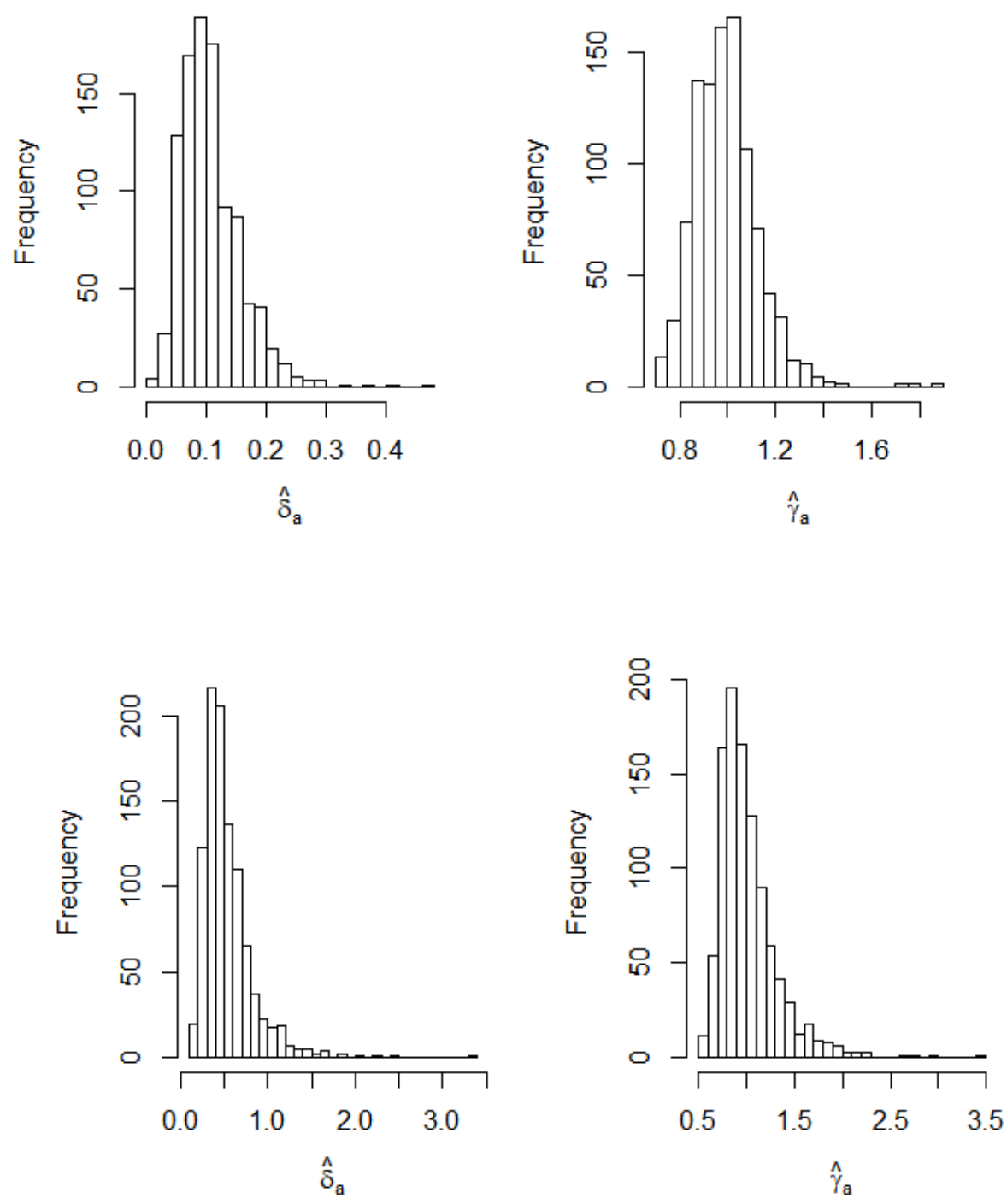

Figure 1. Histogram for estimates in Table 2 for the Fréchet simulation corresponding to $\delta_{0}=0.1$ (top) and $\delta_{0}=0.5$ (bottom).

distributions with index $\alpha=1.0$. In all cases, we take $k_{n}=200$ and begin by maximizing the bivariate likelihood to obtain initial estimates of $\delta_{0}$ and, more importantly, $\gamma_{0}$. The method of Section 3 is used throughout.

(i) no missing extremes. In this case, no extremes have been removed from the simulated data so that $\delta_{0}=0$. The estimates of $\delta_{0}$ and $\gamma_{0}$ using the method of Section 3 with $m=10$ are near 0 and 0.912 in the Pareto case, and 0.001 and 0.904 in the Fréchet case. In order to test our estimation procedure, for each $\delta_{i}=i / k_{n}, i=1, \ldots, k_{n}$, we remove 
the upper $i$ extremes of the simulated data set and then compute $\hat{\delta}_{i}$, by minimizing the objective function (3.5) for fixed values of $\gamma=.9,1.0,1.1,1.2$ using the altered data, i.e., with the appropriate number of extremes removed. (As mentioned above, we avoid here maximizing the bivariate likelihood and, at the same time, check the robustness of the univariate likelihood maximization to a mild misspecification of $\gamma$.) In Figure 2 we plot $\hat{\delta}$ vs. $\delta$ for each of the the four choices of $\gamma$ for the Pareto sample (left panel) and the Fréchet sample (right panel). Notice that each of the four curves are approximately linear with intercept 0 , strongly suggesting that $\delta_{0}=0$. The red line (corresponding to the true $\gamma=1$ ) has the slope closest to 1 .
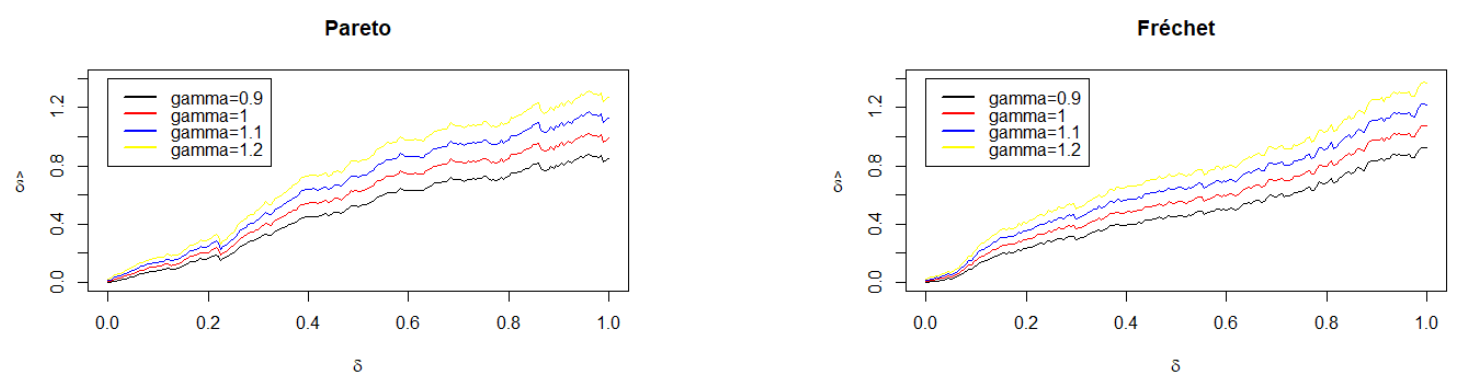

Figure 2. Estimated number of missing extremes for samples from Pareto and Fréchet distributions with $n=5000, k_{n}=200, \gamma_{0}=1$.

(ii) the upper $\delta_{0} k_{n}$ extremes are missing. For this simulation, we take $\delta_{0}=.25$ so that 50 largest observations are removed from the samples described in (i). The estimates of $\delta_{0}$ and $\gamma_{0}$ are 0.145 and 0.769 for the Pareto case and 0.431 and 1.122 for the Fréchet case. The same style plots as those in Figure 2 are displayed in Figure 3. The reader should keep in mind that now the horizontal axis for $\delta$ corresponds to $\delta k_{n}$ extreme observations missing from the observed data (in addition to the 50 largest removed from the originally generated data). The plots of $\hat{\delta}$ vs $\delta$ are again nearly linear for the four values of $\gamma$, with the red line $(\gamma=1)$ having slope closest to 1 , and the corresponding intercept value close to the true $\delta_{0}=.25$. 

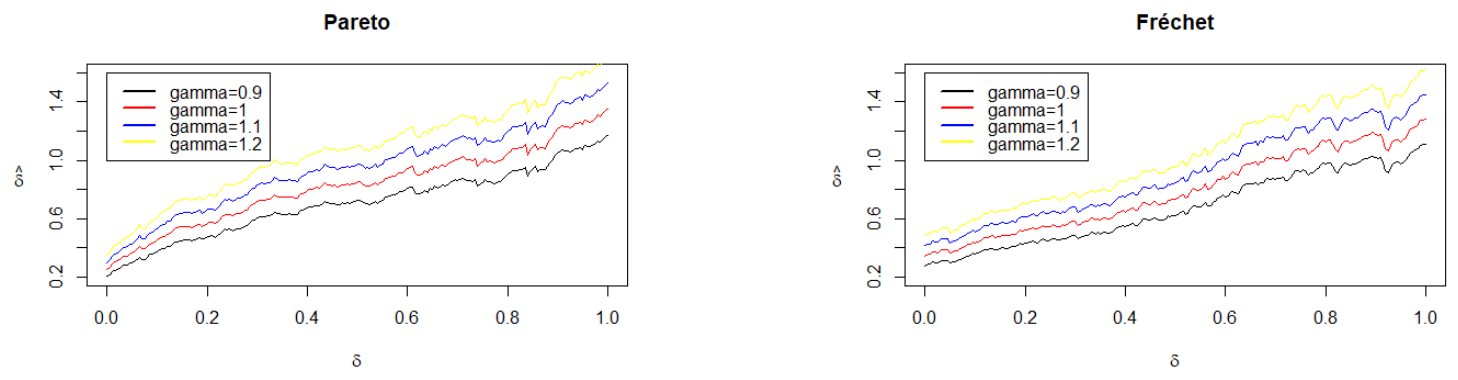

Figure 3. Estimated number of missing extremes for samples from Pareto and Fréchet distributions with the 50 largest observations removed. $n=$ $5000, k_{n}=200, \gamma_{0}=1$.

(iii) the $\delta_{0} k_{n}$ missing extremes are not consecutive. For this simulation, we again take $\delta_{0}=.25$, but this time the 50 missing extremes are randomly selected from among the 100 largest observations. The estimate of $\delta_{0}$ is near 0 and the estimate of $\gamma_{0}$ is 0.774 in the Pareto case, while the estimates of $\delta_{0}$ and $\gamma_{0}$ are 0.004 and 0.789 in the Fréchet case. For this scenario $\delta^{\dagger}=50 / 200=.25$ so that after removing another 50 extremes from the observed data, we have all 100 of the top extremes removed. The corresponding plots displayed in Figure 4 now have a different look. They are essentially connected segments with nodes around $\delta$ just over .1 and just over .2 for both the Pareto and Fréchet cases, respectively. Notice that locations of these nodes are robust to the choice of $\gamma$; each of the 4 curves have nodes at approximately the same horizontal location. On the Pareto plot we would estimate $\delta^{\dagger}$ to be around .24, which is near the true $\delta^{\dagger}$ of .25 . The third segment of the red curve (corresponding to the true $\gamma=1$ ), from .24 to 1 , has the slope closest to 1 . For this curve the value at .24 is .63 . This gives us an estimate of $\delta_{0}$ as $.63-.24=.39$. A similar analysis for the Fréchet case gives us an estimate of $\delta^{\dagger}=.22$ with corresponding value on the red curve of .54 . The estimate of $\delta_{0}$ is then $.54-.22=.32$. So in both cases, we retrieve reasonable (though not perfect) estimates of $\delta_{0}=.25$.
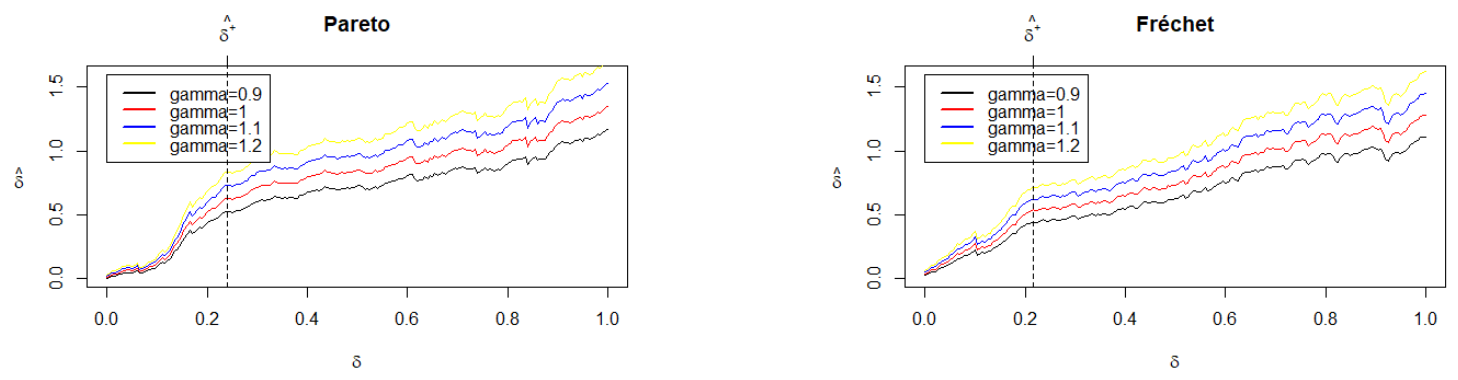

FIGURE 4. Estimated number of missing extremes and tail index for samples from Pareto and Fréchet distributions, with 50 observations among the largest 100, removed. $n=5000, k_{n}=200, \gamma_{0}=1$. 


\section{Applications}

In this section, we apply the methodology described in Sections 5.1 and 5.2 to several real data sets. The goal here is to estimate the shape parameter $\gamma_{0}$ in addition to $\delta_{0}$ and $\delta^{\dagger}$, where $\delta_{0} k_{n}$ is the number of missing extremes among the largest $\left(\delta^{\dagger}+\delta_{0}\right) k_{n}$ extremes. Of course if our estimate is $\hat{\delta}^{\dagger}=0$, we declare that missing extremes, if any, are consecutive. Once again, the estimation method of Section 3 is used throughout.

6.1. Danish Fire Insurance. The Danish Fire Insurance data set is a standard example used in extreme value theory. It is a part of the R-statistics package and consists of 2492 large Danish fire insurance claims from January 1, 1980 to December 31, 1990. Using $k_{n}=50$ and $m=10$, the estimate of $\delta_{0}$ is near 0 and the estimate of $\gamma_{0}$ is 0.565 . Next we explore the possibility of some missing (not necessarily consecutive) extremes by applying the methodology in Section 5.2 with 4 values of $\gamma$ based on the initial estimate. The resulting plots of $\hat{\delta}$ vs $\delta$ for four different values of $\gamma$ are displayed in Figure 5. All of these plots look roughly linear without any obvious nodes, with the intercepts close to 0 . Hence we estimate $\hat{\delta}^{\dagger}=0=\hat{\delta}_{0}$. The blue curve appears to have the slope closest to 1 , so we estimate $\hat{\gamma}_{0}$ to be around .7 .

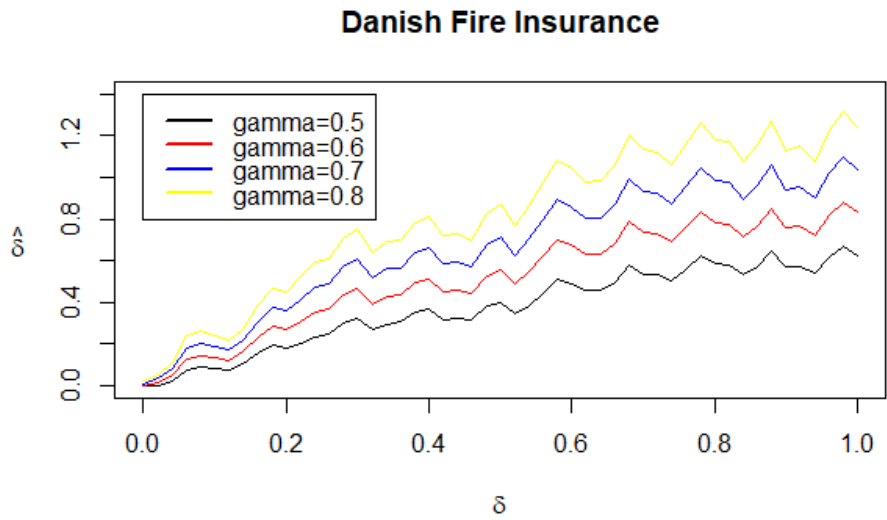

FiguRE 5. Estimated number of missing extremes for Danish fire insurance. $n=2492, k_{n}=50$.

6.2. Google+. The second example consists of the in-degrees values from 76,438,791 nodes in a snapshot of the Google+ social network that was explored in Zou et al. (2019). They concluded that around 150 consecutive largest extremes were missing. Using $k_{n}=500$, the methodology of Section 3 gives initial estimates of $\delta_{0}$ and $\gamma_{0}$ as .327 and 1.418, respectively, so that the number of missing would be $.327 * 500=163$ missing extremes. This estimate is consistent with the number of missing found in Zou et al. (2019). Unfortunately, our estimate of $\gamma_{0}$ does not produce reasonable plots as described in Section 5.2, so upon further experimentation we settled on a different range of $\gamma$ and construct plots of $\hat{\delta}$ by removing the largest observations for four values of $\gamma=3,3.5,4,4.5$. The resulting plots are displayed in Figure 6. Using the methodology of Section 5.2, we would estimate $\hat{\delta}^{\dagger}$ to be near 0.036 , and the red curve appears to have slope closest to 1 . We therefore estimate 
$\gamma_{0}$ to be close to 3.5. The value of the red curve corresponding to $\hat{\delta}^{\dagger}$ is 1.38 , so we estimate $\delta_{0}$ as $1.38-.036=1.344$. That corresponds to $1.344 * 500=672$ missing extremes among $1.38 * 500=690$ largest extremes. We now remove the additional $.036 * 500=18$ largest values in the data set and re-plot in Figure 7, the curves corresponding to the 4 values of $\gamma$ above. Note that all curves are roughly linear and that the red curve $(\gamma=3.5)$ has the slope closest to 1 .

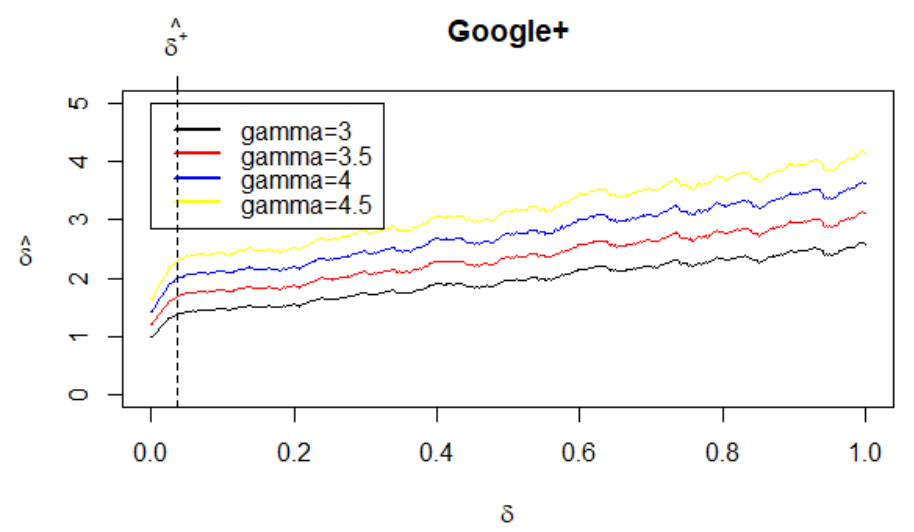

FIgURE 6. Estimated number of missing extremes for Google + with $k_{n}=$ 500, with $\hat{\delta}^{\dagger}=0.036$ marked.

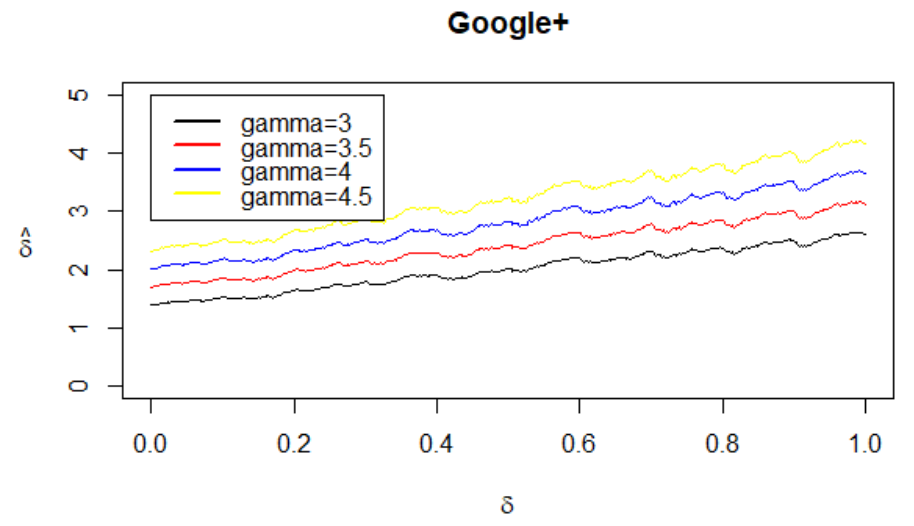

Figure 7. Estimated number of missing extremes for Google + with the 18 largest observations removed, $k_{n}=500$.

6.3. Natural and Climate Disasters in the U.S. from 1980-2019. This data, which can be accessed from http://ncdc.noaa.gov/billions/, was assembled by the National Oceanic and Atmospheric Administration (NOAA). They identify 258 costly natural and climatic events such as wild fires, hurricanes, flooding, earthquakes, droughts, tornadoes, and severe 
storms during the period from January 1980 through December 2019. This data set represents the financial costs in billions of 2019 US dollars associated with these events. More details about the data set can be found in Smith and Katz (2013). While one should always exercise caution in applying extreme value theory to small data sets, we nevertheless apply our methods in this case with $k_{n}=30$. The initial estimates of $\delta_{0}$ and $\gamma_{0}$ are .08 and 1.349, respectively. This would lead to an estimate of $0.08 * 30=2.4$ missing extremes. Since there are no truly missing extremes (every disaster event has a recorded value), we interpret missingness as being reflective of some extremes being underreported. As done previously, we explore the possibility that there are non-consecutive missing extremes among of a fraction of the largest observations. To this end, we construct plots of $\hat{\delta}$ by removing largest observations for four values of $\gamma=1.3,1.4,1.5,1.6$. The resulting plots are in Figure 8 . As in Section 5.2, the estimate $\hat{\delta}^{\dagger}$ is near 0.667, and the red curve has the slope closest to 1 in the last part of the plot. We therefore estimate $\gamma_{0}$ to be near 1.4. The value of the red curve at $\hat{\delta}^{\dagger}$ is 1.193 , so we estimate $\delta_{0}$ as $1.193-0.667=0.526$, corresponding to 16 missing extremes. Now re-estimating $\delta_{0}$ and $\gamma_{0}$ for the observed data with the additional $\hat{\delta}^{\dagger} k_{n}=0.667 * 30=20$ extreme observations removed, we obtain $\hat{\delta}_{0}=1.193$ and $\hat{\gamma}_{0}=1.718$. So our final estimate of $\delta_{0}$ would be $1.193-0.667=0.526$. This corresponds to $0.526 * 30=16$ missing observations among the $1.193 * 30=36$ largest extremes. After having removed $36-16=20$ largest values from the data set and re-plotting the curves corresponding to the four value of $\gamma$ above, we see the results in Figure 9. The curves are roughly linear, with the red curve having the slope closest to 1.

Natural and Climate Disasters in the U.S. from 1980-2019

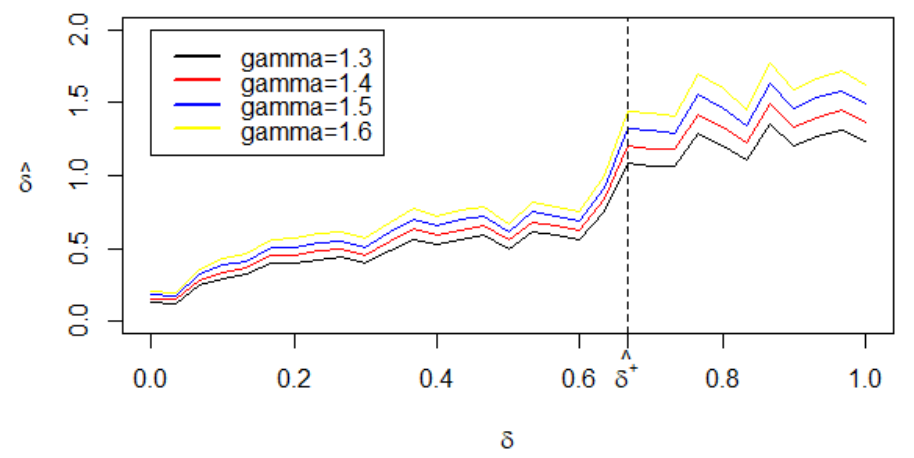

Figure 8. Weather and climate disasters from 1980 to 2019. $n=258, k_{n}=30$.

\section{APPENDIX}

7.1. Second-order regular variation. Second-order regular variation can be thought of as a way to quantify the vanishing difference between the left hand side and the right hand side of (1.1). It assumes that there is $\rho \leq 0$ and a positive or negative function $A$ that is 
Natural and Climate Disasters in the U.S. from 1980-2019

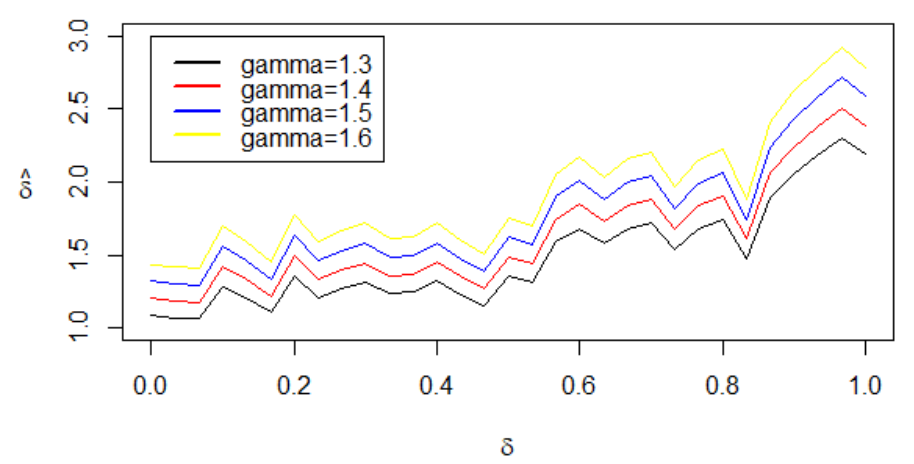

FiguRE 9. Weather and climate disasters from 1980 to 2019 with 20 largest observations removed. $n=258, k_{n}=30$.

regularly varying with exponent $\rho$ and $\lim _{t \rightarrow \infty} A(t)=0$, such that for $x>0$,

$$
\lim _{t \rightarrow \infty} \frac{\log U(t x)-\log U(t)-\gamma \log x}{A(t)}=\left\{\begin{array}{cc}
\frac{x^{\rho}-1}{\rho} & \rho<0 \\
\log x & \rho=0
\end{array}\right.
$$

where $U(t)=F^{\leftarrow}(1-1 / t)$ and $F^{\leftarrow}$ is the generalized inverse of $F$; see e.g. de Haan and Ferreira (2006).

The results of this paper assume that the sequence $\left(k_{n}\right)$ used to define our estimators satisfies

$$
\lim _{n \rightarrow \infty} \sqrt{k_{n}} A\left(n / k_{n}\right)=\lambda
$$

for some $\lambda \in \mathbb{R}$. Since $k_{n} \rightarrow \infty$, condition (7.2) implies that $n / k_{n} \rightarrow \infty$.

Distributions that satisfy the second-order condition include the Student's $t_{\nu}$, stable, and Fréchet distributions; see, e.g. Drees (1998) and Drees et al. (2000). In fact, any distribution with $\bar{F}(x)=c_{1} x^{-\alpha}+c_{2} x^{-\alpha+\alpha \rho}(1+o(1))$ as $x \rightarrow \infty$, where $c_{1}>0, c_{2} \neq 0$, $\alpha>0$ and $\rho<0$, satisfies the second-order condition with the indicated values of $\alpha$ and $\rho$ (de Haan and Ferreira (2006)).

7.2. Proofs. In this section we present the proofs of the results in the earlier parts of the paper.

Proof of Lemma 3.1. Since

$$
\partial_{1} L_{n}\left(\gamma_{0}, \delta_{0}\right)=\frac{2 m}{\gamma_{0}}-\frac{2}{\gamma_{0}} \sum_{i=1}^{m} \omega_{i, \delta_{0}} Y_{n i}^{2}-\frac{2 \sqrt{k_{n}}}{\gamma_{0}} \sum_{i=1}^{m} \omega_{i, \delta_{0}} h_{\delta_{0}, i} Y_{n i}
$$

and

$$
\partial_{2} L_{n}\left(\gamma_{0}, \delta_{0}\right)=-\sum_{i=1}^{m} \frac{\omega_{i, \delta_{0}}^{\prime}}{\omega_{i, \delta_{0}}}+\sum_{i=1}^{m} \omega_{i, \delta_{0}}^{\prime} Y_{n i}^{2}-2 \sqrt{k_{n}} \sum_{i=1}^{m} \omega_{i, \delta_{0}} h_{\delta_{0}, i}^{\prime} Y_{n i},
$$

the claim of the lemma follows from (3.3). 
Proof of Lemma 3.2. We proceed as in the proof of Lemma 3.1, except now one needs to take second derivatives. For example, elementary calculations give us

$$
\begin{aligned}
\frac{\partial_{1}^{2} L_{n}(\tilde{\gamma}, \tilde{\delta})}{k_{n}}= & -\frac{2 k}{\tilde{\gamma}^{2} k_{n}}+\frac{6 \gamma_{0}^{2}}{\tilde{\gamma}^{4} k_{n}} \sum_{i=1}^{m} \omega_{i, \tilde{\delta}} Y_{n i}^{2}-\frac{12 \gamma_{0}}{\tilde{\gamma}^{4} \sqrt{k_{n}}} \sum_{i=1}^{m} \omega_{i, \tilde{\delta}}\left(\tilde{\gamma} h_{\tilde{\delta, i}}-\gamma_{0} h_{\delta_{0}, i}\right) Y_{n i} \\
& +\frac{6}{\tilde{\gamma}^{4}} \sum_{i=1}^{m} \omega_{i, \tilde{\delta}}\left(\tilde{\gamma} h_{\tilde{\delta, i}}-\gamma_{0} h_{\delta_{0}, i}\right)^{2}+\frac{8 \gamma_{0}}{\tilde{\gamma}^{3} \sqrt{k_{n}}} \sum_{i=1}^{m} \omega_{i, \tilde{\delta}} h_{\tilde{\delta, i}} Y_{n i} \\
& -\frac{8}{\tilde{\gamma}^{3}} \sum_{i=1}^{m} \omega_{i, \tilde{\delta}} h_{\tilde{\delta}, i}\left(\tilde{\gamma} h_{\tilde{\delta, i}}-\gamma_{0} h_{\delta_{0}, i}\right)+\frac{2}{\tilde{\gamma}^{2}} \sum_{i=1}^{m} \omega_{i, \tilde{\delta}} h_{\tilde{\delta, i}}^{2} .
\end{aligned}
$$

Using (3.3) and the fact that $(\tilde{\gamma}, \tilde{\delta}) \stackrel{P}{\rightarrow}\left(\gamma_{0}, \delta_{0}\right)$ we see that

$$
\frac{\partial_{1}^{2} L_{n}(\tilde{\gamma}, \tilde{\delta})}{k_{n}} \stackrel{P}{\rightarrow} \frac{2}{\gamma_{0}^{2}} \sum_{i=1}^{m} \omega_{i, \delta_{0}} h_{\delta_{0}, i}^{2}=\frac{2 b_{m}}{\gamma_{0}^{2}} .
$$

The other terms of the Hessian matrix can be handled in a similar manner.

Proof of Lemma 3.3. Denote

$$
L(\gamma, \delta)=\gamma^{-2} \sum_{i=1}^{m} \omega_{i, \delta}\left(\gamma h_{\delta, i}-\gamma_{0} h_{\delta_{0}, i}\right)^{2}, \quad(\gamma, \delta) \in \Theta .
$$

Since we can write

$$
\begin{aligned}
L_{n}(\gamma, \delta) / k_{n} & =\frac{2 m \log \gamma}{k_{n}}-\frac{1}{k_{n}} \sum_{i=1}^{m} \log \omega_{i, \delta}+\frac{\gamma_{0}^{2}}{\gamma^{2} k_{n}} \sum_{i=1}^{m} \omega_{i, \delta} Y_{n i}^{2} \\
& -\frac{2 \gamma_{0}}{\gamma^{2} \sqrt{k_{n}}} \sum_{i=1}^{m} \omega_{i, \delta}\left(\gamma h_{\delta, i}-\gamma_{0} h_{\delta_{0}, i}\right) Y_{n i}+\frac{1}{\gamma^{2}} \sum_{i=1}^{m} \omega_{i, \delta}\left(\gamma h_{\delta, i}-\gamma_{0} h_{\delta_{0}, i}\right)^{2},
\end{aligned}
$$

we have

$$
\begin{aligned}
& \sup _{(\gamma, \delta) \in \Theta}\left|\frac{L_{n}(\gamma, \delta)}{k_{n}}-L(\gamma, \delta)\right| \\
\leq & \sup _{(\gamma, \delta) \in \Theta}\left|\frac{2 m \log \gamma}{k_{n}}-\frac{1}{k_{n}} \sum_{i=1}^{m} \log \omega_{i, \delta}\right|+\sup _{(\gamma, \delta) \in \Theta}\left|\frac{\gamma_{0}^{2}}{\gamma^{2} k_{n}} \sum_{i=1}^{m} \omega_{i, \delta} Y_{n i}^{2}\right| \\
+ & \sup _{(\gamma, \delta) \in \Theta}\left|\frac{2 \gamma_{0}}{\gamma^{2} \sqrt{k_{n}}} \sum_{i=1}^{m} \omega_{i, \delta}\left(\gamma h_{\delta, i}-\gamma_{0} h_{\delta_{0}, i}\right) Y_{n i}\right| \stackrel{P}{\rightarrow} 0, \quad n \rightarrow \infty,
\end{aligned}
$$

by (3.3), since we know that, by assumption, $\gamma, \omega_{i, \delta}$ and $h_{\delta, i}$ are bounded away from 0 and infinity on $\Theta$.

Clearly, the point $\left(\gamma_{0}, \delta_{0}\right)$ is a minimizer of the function $\gamma^{2} L$. Furthermore, it is elementary to check that the Hessian matrix of $\gamma^{2} L$ at that point is equal to $2 \gamma_{0}^{2} \Gamma_{m}$. We will see in the proof of Proposition 3.1 below that the matrix $\Gamma_{m}$ is invertible, hence the point $\left(\gamma_{0}, \delta_{0}\right)$ is the unique minimizer of the function $\gamma^{2} L$, hence also of the function $L$. The uniform convergence in probability of the function $L_{n} / k_{n}$ to the function $L$ implies that any minimizer of the former function converges in probability to the unique minimizer of the limit function. Hence the statement of the lemma. 
Proof of Proposition 3.1. Introduce functions of $x>0$

$$
l_{\delta}(x)=x^{2} /(x+\delta), \quad m_{\delta}(x)=x^{2} v(x / \delta) / \delta=x-2 \delta \log (1+x / \delta)+\delta x /(x+\delta),
$$

so that

$$
\begin{aligned}
\omega_{i, \delta_{0}} & =\theta_{i}^{2} /\left(m_{\delta_{0}}\left(\theta_{i}\right)-m_{\delta_{0}}\left(\theta_{i-1}\right)\right), \quad g_{\delta_{0}, i}=\left(m_{\delta_{0}}\left(\theta_{i}\right)+l_{\delta_{0}}\left(\theta_{i}\right)\right) / 2 \theta_{i}, \\
g_{\delta_{0}, i}^{\prime} & =\left(m_{\delta_{0}}\left(\theta_{i}\right)-l_{\delta_{0}}\left(\theta_{i}\right)\right) / 2 \delta_{0} \theta_{i}, \quad i=1, \ldots, m .
\end{aligned}
$$

Therefore we can write

$$
\begin{aligned}
b_{m} & =\frac{m_{\delta_{0}}\left(\theta_{m}\right)+2 l_{\delta_{0}}\left(\theta_{m}\right)}{4}+\frac{1}{4} \sum_{i=1}^{m} \frac{\left(l_{\delta_{0}}\left(\theta_{i}\right)-l_{\delta_{0}}\left(\theta_{i-1}\right)\right)^{2}}{m_{\delta_{0}}\left(\theta_{i}\right)-m_{\delta_{0}}\left(\theta_{i-1}\right)} \\
c_{m} & =\frac{m_{\delta_{0}}\left(\theta_{m}\right)-2 l_{\delta_{0}}\left(\theta_{m}\right)}{4 \delta_{0}^{2}}+\frac{1}{4 \delta_{0}^{2}} \sum_{i=1}^{m} \frac{\left(l_{\delta_{0}}\left(\theta_{i}\right)-l_{\delta_{0}}\left(\theta_{i-1}\right)\right)^{2}}{m_{\delta_{0}}\left(\theta_{i}\right)-m_{\delta_{0}}\left(\theta_{i-1}\right)}, \\
d_{m} & =\frac{m_{\delta_{0}}\left(\theta_{m}\right)}{4 \delta_{0}}-\frac{1}{4 \delta_{0}} \sum_{i=1}^{m} \frac{\left(l_{\delta_{0}}\left(\theta_{i}\right)-l_{\delta_{0}}\left(\theta_{i-1}\right)\right)^{2}}{m_{\delta_{0}}\left(\theta_{i}\right)-m_{\delta_{0}}\left(\theta_{i-1}\right)}
\end{aligned}
$$

We now show that the matrix $\Gamma_{m}$ is invertible. A direct computation shows that

$$
4 \delta_{0}^{2}\left(b_{m} c_{m}-d_{m}^{2}\right)=m_{\delta_{0}}\left(\theta_{m}\right) \sum_{i=1}^{m} \frac{\left(l_{\delta_{0}}\left(\theta_{i}\right)-l_{\delta_{0}}\left(\theta_{i-1}\right)\right)^{2}}{m_{\delta_{0}}\left(\theta_{i}\right)-m_{\delta_{0}}\left(\theta_{i-1}\right)}-l_{\delta_{0}}^{2}\left(\theta_{m}\right) .
$$

It is easy to check that the functions $l_{\delta_{0}}$ and $m_{\delta_{0}}$ are increasing on $(0, \infty)$, so that for any $i \geq 1, l_{\delta_{0}}\left(\theta_{i}\right)-l_{\delta_{0}}\left(\theta_{i-1}\right)>0$ and $m_{\delta_{0}}\left(\theta_{i}\right)-m_{\delta_{0}}\left(\theta_{i-1}\right)>0$. Further, by the Cauchy-Schwarz inequality,

$$
\begin{aligned}
m_{\delta_{0}}\left(\theta_{m}\right) \sum_{i=1}^{m} \frac{\left(l_{\delta_{0}}\left(\theta_{i}\right)-l_{\delta_{0}}\left(\theta_{i-1}\right)\right)^{2}}{m_{\delta_{0}}\left(\theta_{i}\right)-m_{\delta_{0}}\left(\theta_{i-1}\right)} & =\sum_{i=1}^{m}\left(m_{\delta_{0}}\left(\theta_{i}\right)-m_{\delta_{0}}\left(\theta_{i-1}\right)\right) \sum_{i=1}^{m} \frac{\left(l_{\delta_{0}}\left(\theta_{i}\right)-l_{\delta_{0}}\left(\theta_{i-1}\right)\right)^{2}}{m_{\delta_{0}}\left(\theta_{i}\right)-m_{\delta_{0}}\left(\theta_{i-1}\right)} \\
& \geq\left(\sum_{i=1}^{m}\left(l_{\delta_{0}}\left(\theta_{i}\right)-l_{\delta_{0}}\left(\theta_{i-1}\right)\right)\right)^{2}=l_{\delta_{0}}^{2}\left(\theta_{m}\right),
\end{aligned}
$$

and the equality holds if and only if

$$
\frac{l_{\delta_{0}}\left(\theta_{1}\right)}{m_{\delta_{0}}\left(\theta_{1}\right)}=\frac{l_{\delta_{0}}\left(\theta_{2}\right)-l_{\delta_{0}}\left(\theta_{1}\right)}{m_{\delta_{0}}\left(\theta_{2}\right)-m_{\delta_{0}}\left(\theta_{1}\right)}=\cdots=\frac{l_{\delta_{0}}\left(\theta_{m}\right)-l_{\delta_{0}}\left(\theta_{m-1}\right)}{m_{\delta_{0}}\left(\theta_{m}\right)-m_{\delta_{0}}\left(\theta_{m-1}\right)} .
$$

The latter requirement is equivalent to

$$
\frac{l_{\delta_{0}}\left(\theta_{1}\right)}{m_{\delta_{0}}\left(\theta_{1}\right)}=\frac{l_{\delta_{0}}\left(\theta_{2}\right)}{m_{\delta_{0}}\left(\theta_{2}\right)}=\cdots=\frac{l_{\delta_{0}}\left(\theta_{m}\right)}{m_{\delta_{0}}\left(\theta_{m}\right)},
$$

so invertibility of $\Gamma_{m}$ will follow once we show that (7.3) cannot hold. If we put

$$
Q(x)=: m_{\delta_{0}}(x)-\frac{l_{\delta_{0}}(x) m_{\delta_{0}}^{\prime}(x)}{l_{\delta_{0}}^{\prime}(x)}=m_{\delta_{0}}(x)-\frac{x^{3}}{\left(x+\delta_{0}\right)\left(x+2 \delta_{0}\right)},
$$

then $Q(0)=0$ and

$$
Q^{\prime}(x)=-\frac{2 \delta_{0} x^{2}}{\left(x+\delta_{0}\right)\left(x+2 \delta_{0}\right)^{2}}<0, \quad x>0,
$$


which implies that

$$
Q(x)=\frac{l_{\delta_{0}}^{\prime}(x)}{m_{\delta_{0}}^{2}(x)}\left(\frac{l_{\delta_{0}}(x)}{m_{\delta_{0}}(x)}\right)^{\prime}<0
$$

for any $x>0$. Since $l_{\delta_{0}}^{\prime}(x)>0$, we conclude that the function $l_{\delta_{0}}(x) / m_{\delta_{0}}(x)$ is strictly decreasing on the positive half line, and so (7.3) cannot hold. Hence the matrix $\Gamma_{m}$ is invertible.

It is elementary to check that, as $\delta_{0} \rightarrow 0$,

$$
b_{m} \rightarrow \theta_{m}, \quad c_{m} \sim \theta_{1}^{-1}\left(\log \delta_{0}\right)^{2}, \quad d_{m} \rightarrow \log \delta_{0} .
$$

Substituting this into (3.8) shows convergence of the variance in (3.10).

Similarly, it is elementary to check that, as $\delta_{0} \rightarrow 0$,

$$
a_{1} \rightarrow \frac{2 \lambda}{1-\rho} \gamma_{0}^{-2} \theta_{m}^{1-\rho}, \quad a_{2} \sim \frac{2 \lambda}{1-\rho} \gamma_{0}^{-1} \theta_{1}^{-\rho} \log \delta_{0} .
$$

Substituting (7.4) and (7.5) into (3.8) and (3.9) proves convergence of the mean in (3.10).

Proof of Lemma 4.1. By (4.2),

$$
\partial_{1} L_{n}\left(\gamma_{0}, \delta_{0}\right)=\frac{2 k_{n}}{\gamma_{0}}-\frac{2 \omega_{1, \delta_{0}} \eta_{n}^{2}}{\gamma_{0}}-\frac{2 \sqrt{k_{n}}}{\gamma_{0}} \omega_{1, \delta_{0}} g_{\delta_{0}, 1} \eta_{n}-\frac{2}{\gamma_{0}} \sum_{i=2}^{k_{n}} Z_{i, n}
$$

and

$$
\partial_{2} L_{n}\left(\gamma_{0}, \delta_{0}\right)=-\frac{\omega_{1, \delta_{0}}^{\prime}}{\omega_{1, \delta_{0}}}-\sum_{i=2}^{k_{n}} \frac{2}{\delta_{0}+\theta_{i, n}}+\omega_{1, \delta_{0}}^{\prime} \eta_{n}^{2}-2 \sqrt{k_{n}} \omega_{1, \delta_{0}} g_{\delta_{0}, 1}^{\prime} \eta_{n}+2 \sum_{i=2}^{k_{n}} \frac{Z_{i, n}}{\delta_{0}+\theta_{i, n}} .
$$

Since

$$
\left(\begin{array}{c}
-\frac{2}{\gamma_{0}} \omega_{1, \delta_{0}} g_{\delta_{0}, 1} \eta_{n} \\
-2 \omega_{1, \delta_{0}} g_{\delta_{0}, 1}^{\prime} \eta_{n}
\end{array}\right) \Rightarrow N\left(0,4 \Gamma_{1}\right)
$$

the claim of the lemma will follow once we show that

$$
\left(\begin{array}{c}
-k_{n}^{-1 / 2} \gamma_{0}^{-1} \sum_{i=2}^{k_{n}}\left(Z_{i, n}-1\right) \\
k_{n}^{-1 / 2} \sum_{i=2}^{k_{n}} \frac{Z_{i, n}-1}{\delta_{0}+\theta_{i, n}}
\end{array}\right) \Rightarrow N\left(0, \Gamma_{0}\right)
$$

where $\Gamma_{0}$ is the second matrix in the right hand side of (4.3). By (4.1) we only need to prove that

$$
\left(\begin{array}{c}
-k_{n}^{-1 / 2} \gamma_{0}^{-1} \sum_{i=2}^{k_{n}} \frac{k_{n}\left(\delta_{0}+\theta_{i, n}\right)}{\left[\delta_{0} k_{n}\right]+\left[\theta_{i, n} k_{n}\right]}\left(E_{\left[\delta_{0} k_{n}\right]+\left[\theta_{i, n} k_{n}\right]}^{*}-1\right) \\
k_{n}^{-1 / 2} \sum_{i=2}^{k_{n}} \frac{k_{n}}{\left[\delta_{0} k_{n}\right]+\left[\theta_{i, n} k_{n}\right]}\left(E_{\left[\delta_{0} k_{n}\right]+\left[\theta_{i, n} k_{n}\right]}^{*}-1\right)
\end{array}\right) \Rightarrow N\left(0, \Gamma_{0}\right) .
$$

Since the covariance matrix the random vector in the right hand side of (7.6) converges to $\Gamma_{0}$, only the Lyapunov condition needs to be checked for an application of the central limit theorem. The latter can be performed component-wise and is elementary when taking, for instance, the 4 th powers of the terms.

Proof of Lemma 4.2. Once again, computing the second derivatives, we obtain, for example,

$$
\frac{\partial_{1}^{2} L_{n}(\tilde{\gamma}, \tilde{\delta})}{k_{n}}=-\frac{2}{\tilde{\gamma}^{2}}+\frac{6 \omega_{1, \tilde{\delta}}}{\tilde{\gamma}^{4} k_{n}}\left(\gamma_{0} \eta_{n}-\sqrt{k_{n}}\left(\tilde{\gamma} g_{\tilde{\delta}, 1}-\gamma_{0} g_{\delta_{0}, 1}\right)\right)^{2}
$$




$$
\begin{aligned}
& +\frac{8 \omega_{1, \tilde{\delta}} g_{\tilde{\delta}, 1}}{\tilde{\gamma}^{3} \sqrt{k_{n}}}\left(\gamma_{0} \eta_{n}-\sqrt{k_{n}}\left(\tilde{\gamma} g_{\tilde{\delta}, 1}-\gamma_{0} g_{\delta_{0}, 1}\right)\right)+\frac{2}{\tilde{\gamma}^{2}} \omega_{1, \tilde{\delta}} g_{\tilde{\delta}, 1}^{2} \\
& +\frac{4 \gamma_{0}}{\tilde{\gamma}^{3} k_{n}} \sum_{i=2}^{k_{n}} \frac{\left(\tilde{\delta}+\theta_{i, n}\right) Z_{i, n}}{\delta_{0}+\theta_{i, n}} .
\end{aligned}
$$

Clearly, the second and the third terms in the right hand side are $o_{p}(1)$ as $n \rightarrow \infty$. Furthermore,

$$
-\frac{2}{\tilde{\gamma}^{2}} \rightarrow-\frac{2}{\gamma_{0}^{2}}, \quad \frac{2}{\tilde{\gamma}^{2}} \omega_{1, \tilde{\delta}} g_{\tilde{\delta}, 1}^{2} \rightarrow \frac{2}{\gamma_{0}^{2}} \omega_{1, \delta_{0}} g_{\delta_{0}, 1}^{2} \text { in probability, }
$$

and by computing the mean and the variance we see that

$$
\frac{4 \gamma_{0}}{\tilde{\gamma}^{3} k_{n}} \sum_{i=2}^{k_{n}} \frac{\left(\tilde{\delta}+\theta_{i, n}\right) Z_{i, n}}{\delta_{0}+\theta_{i, n}} \rightarrow \frac{4}{\gamma_{0}^{2}}
$$

in probability. Therefore,

$$
\frac{\partial_{1}^{2} L_{n}(\tilde{\gamma}, \tilde{\delta})}{k_{n}} \rightarrow \frac{2}{\gamma_{0}^{2}}+\frac{2}{\gamma_{0}^{2}} \omega_{1, \delta_{0}} g_{\delta_{0}, 1}^{2}
$$

in probability, and the limit is the appropriate entry in the matrix $2 \Gamma_{\infty}$. The other terms of the Hessian matrix can be handled in a similar manner.

Proof of Lemma 4.3. We proceed as in the proof of Lemma 3.3. Denote now

$$
L(\gamma, \delta)=2 \log \gamma+\frac{\tilde{\omega}_{1, \delta}}{\gamma^{2}}\left(\gamma \tilde{g}_{\delta, 1}-\gamma_{0} \tilde{g}_{\delta_{0}, 1}\right)^{2}-2 \int_{\varepsilon}^{1+\varepsilon} \log (\delta+x) d x+\frac{2 \gamma_{0}}{\gamma} \int_{\varepsilon}^{1+\varepsilon} \frac{\delta+x}{\delta_{0}+x} d x,
$$

where $\tilde{\omega}_{1, \delta}$ is defined as $\omega_{1, \delta}$ and $\tilde{g}_{\delta, 1}$ is defined as $g_{\delta, 1}$, both with $\theta_{1}=\varepsilon$. Since we can write

$$
\begin{aligned}
L_{n}(\gamma, \delta) / k_{n} & =2 \log \gamma-\frac{1}{k_{n}} \log \omega_{1, \delta}-\frac{2}{k_{n}} \sum_{i=2}^{k_{n}} \log \left(\delta+\theta_{i, n}\right)+\frac{\gamma_{0}^{2} \omega_{1, \delta}}{\gamma^{2} k_{n}} \eta_{n}^{2} \\
& -\frac{2 \gamma_{0} \omega_{1, \delta}}{\gamma^{2} \sqrt{k_{n}}}\left(\gamma g_{\delta, 1}-\gamma_{0} g_{\delta_{0}, 1}\right) \eta_{n}+\frac{\omega_{1, \delta}}{\gamma^{2}}\left(\gamma g_{\delta, 1}-\gamma_{0} g_{\delta_{0}, 1}\right)^{2}+\frac{2 \gamma_{0}}{\gamma k_{n}} \sum_{i=2}^{k_{n}} \frac{\left(\delta+\theta_{i, n}\right) Z_{i, n}}{\delta_{0}+\theta_{i, n}},
\end{aligned}
$$

it follows that

$$
\begin{aligned}
& \sup _{(\gamma, \delta) \in \Theta}\left|\frac{L_{n}(\gamma, \delta)}{k_{n}}-L(\gamma, \delta)\right| \\
\leq & \sup _{(\gamma, \delta) \in \Theta}\left|\frac{\omega_{1, \delta}}{\gamma^{2}}\left(\gamma g_{\delta, 1}-\gamma_{0} g_{\delta_{0}, 1}\right)^{2}-\frac{\tilde{\omega}_{1, \delta}}{\gamma^{2}}\left(\gamma \tilde{g}_{\delta, 1}-\gamma_{0} \tilde{g}_{\delta_{0}, 1}\right)^{2}\right| \\
+ & \sup _{(\gamma, \delta) \in \Theta}\left|-\frac{1}{k_{n}} \log \omega_{1, \delta}+\frac{\gamma_{0}^{2} \omega_{1, \delta}}{\gamma^{2} k_{n}} \eta_{n}^{2}-\frac{2 \gamma_{0} \omega_{1, \delta}}{\gamma^{2} \sqrt{k_{n}}}\left(\gamma g_{\delta, 1}-\gamma_{0} g_{\delta_{0}, 1}\right) \eta_{n}\right| \\
+ & \sup _{(\gamma, \delta) \in \Theta}\left|\frac{2}{k_{n}} \sum_{i=2}^{k_{n}} \log \left(\delta+\theta_{i, n}\right)-2 \int_{\varepsilon}^{1+\varepsilon} \log (\delta+x) d x\right| \\
+ & \sup _{(\gamma, \delta) \in \Theta}\left|\frac{2 \gamma_{0}}{\gamma_{k}} \sum_{i=2}^{k_{n}} \frac{\left(\delta+\theta_{i, n}\right) Z_{i, n}}{\delta_{0}+\theta_{i, n}}-\frac{2 \gamma_{0}}{\gamma} \int_{\varepsilon}^{1+\varepsilon} \frac{\delta+x}{\delta_{0}+x} d x\right| .
\end{aligned}
$$


It is clear that the first three terms in the right hand side vanish as $n \rightarrow \infty$. The same is true for the last term in the right hand side because we can bound the latter by

$$
\begin{aligned}
& \sup _{(\gamma, \delta) \in \Theta}\left|\frac{2 \gamma_{0} \delta}{\gamma}\right| \cdot\left|\frac{1}{k_{n}} \sum_{i=2}^{k_{n}} \frac{Z_{i, n}}{\delta_{0}+\theta_{i, n}}-\int_{\varepsilon}^{1+\varepsilon} \frac{1}{\delta_{0}+x} d x\right| \\
+ & \sup _{(\gamma, \delta) \in \Theta}\left|\frac{2 \gamma_{0}}{\gamma}\right| \cdot\left|\frac{1}{k_{n}} \sum_{i=2}^{k_{n}} \frac{\theta_{i, n} Z_{i, n}}{\delta_{0}+\theta_{i, n}}-\int_{\varepsilon}^{1+\varepsilon} \frac{x}{\delta_{0}+x} d x\right| .
\end{aligned}
$$

It is clear that both suprema are finite, while by computing once again the means and the variances we see that the two differences converge to zero in probability.

Clearly, the point $\left(\gamma_{0}, \delta_{0}\right)$ is a minimizer of the function $\tilde{\omega}_{1, \delta} \gamma^{-2}\left(\gamma \tilde{g}_{\delta, 0}-\gamma_{0} \tilde{g}_{\delta_{0}, 1}\right)^{2}$. Let us denote the remaining part of the function $L(\gamma, \delta)$ by $L_{1}(\gamma, \delta)$. To check that the point $\left(\gamma_{0}, \delta_{0}\right)$ is a unique minimizer of the latter function, note that for a fixed value of $\delta$ the unique minimizer of $L_{1}(\cdot, \delta)$ is the point

$$
\gamma(\delta)=\gamma_{0} \int_{\varepsilon}^{1+\varepsilon} \frac{\delta+x}{\delta_{0}+x} d x .
$$

Since, up to $\delta$-independent terms,

$$
L_{1}(\gamma(\delta), \delta)=\log \left(\int_{\varepsilon}^{1+\varepsilon} \frac{\delta+x}{\delta_{0}+x} d x\right)-\int_{\varepsilon}^{1+\varepsilon} \log \left(\frac{\delta+x}{\delta_{0}+x}\right) d x
$$

which vanishes for $\delta=\delta_{0}$ and is strictly positive by Jensen's inequality for $\delta \neq \delta_{0}$, we see that $\delta=\delta_{0}$ and $\gamma=\gamma\left(\delta_{0}=\gamma_{0}\right.$ is the unique minimizer of $L_{1}$ and, hence, also of $L$.

As before, the uniform convergence of $L_{n} / k_{n}$ to $L$ implies now that any minimizer of the former function convergence in probability to $\left(\gamma_{0}, \delta_{0}\right)$. Lemma 4.2 and the fact that $\Gamma_{\infty}$ is invertible mean that, with probability converging to 1 , the minimizer of $L_{n}$ is unique.

\section{REFERENCES}

S. Benchaira, D. Meraghmi and A. Necir (2016): Tail product-limit process for truncated data with application to extreme value index estimation. Extremes 19:219-251.

L. DE HAAn and A. FerReira (2006): Extreme Value Theory: An Introduction. Springer, New York.

H. Drees (1998): On smooth statistical tail functionals. Scandinavian Journal of Statistics 25:187-210.

H. Drees, L. De HaAn and S. Resnick (2000): How to make a Hill plot. Annals of Statistics 28:254-274.

P. Embrechts, C. Klüppelberg and T. Mikosch (1997): Modelling Extremal Events for Insurance and Finance. Springer-Verlag, Berlin.

B. Hill (1975): A simple general approach to inference about the tail of a distribution. Ann. Statist. 3:1163-1174.

M. Newman (2010): Networks: An Introduction. Oxford University Press.

R.-D. ReIss (1989): Asymptotic Distribution of Order Statistics. Springer, New York.

A. B. Smith and R. W. Katz (2013): US billion-dollar weather and climate disasters: data sources, trends, accuracy and biases. Natural hazards 67:387-410.

J. Zou, R. Davis and G. Samorodnitsky (2019): Extreme value analysis without the largest values: what can be done? Probability in the Engineering and Informational Sciences doi:10.1017/S0269964818000542:1-21. 
School of Operations Research and Information Engineering, Cornell University

Email address: hx223@cornell.edu

Department of Statistics, Columbia University

Email address: rdavis@stat.columbia.edu

School of Operations Research and Information Engineering, Cornell University

Email address: gs18@cornell.edu 